ARTICLE

\title{
Graded bulk-heterojunction enables $17 \%$ binary organic solar cells via nonhalogenated open air coating
}

Ying Zhang ${ }^{1}$, Kuan Liu (1) ${ }^{1 凶}$, Jiaming Huang ${ }^{1}$, Xinxin Xia ${ }^{2}$, Jiupeng $\mathrm{Cao}^{3}$, Guangming Zhao (i) ${ }^{3}$,

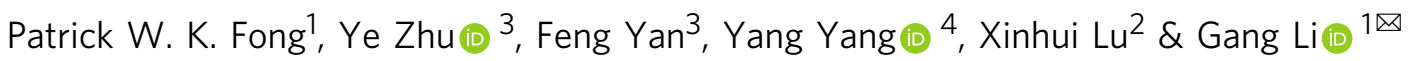

Graded bulk-heterojunction (G-BHJ) with well-defined vertical phase separation has potential to surpass classical BHJ in organic solar cells (OSCs). In this work, an effective G-BHJ strategy via nonhalogenated solvent sequential deposition is demonstrated using nonfullerene acceptor (NFA) OSCs. Spin-coated G-BHJ OSCs deliver an outstanding 17.48\% power conversion efficiency (PCE). Depth-profiling X-ray photoelectron spectroscopy (DP$X P S)$ and angle-dependent grazing incidence $X$-ray diffraction (GI-XRD) techniques enable the visualization of polymer/NFA composition and crystallinity gradient distributions, which benefit charge transport, and enable outstanding thick OSC PCEs $(16.25 \%$ for $300 \mathrm{~nm}$, $14.37 \%$ for $500 \mathrm{~nm}$ ), which are among the highest reported. Moreover, the nonhalogenated solvent enabled G-BHJ OSC via open-air blade coating and achieved a record $16.77 \%$ PCE. The blade-coated G-BHJ has drastically different D-A crystallization kinetics, which suppresses the excessive aggregation induced unfavorable phase separation in $\mathrm{BHJ}$. All these make G-BHJ a feasible and promising strategy towards highly efficient, eco- and manufacture friendly OSCs.

\footnotetext{
${ }^{1}$ Department of Electronic and Information Engineering, Research Institute for Smart Energy (RISE), The Hong Kong Polytechnic University, Hong Kong, China. ${ }^{2}$ Department of Physics, The Chinese University of Hong Kong, Hong Kong, China. ${ }^{3}$ Department of Applied Physics, The Hong Kong Polytechnic University,

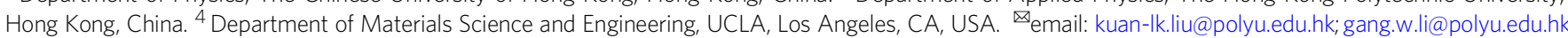


B ulk heterojunction (BHJ) organic solar cells (OSCs) have attracted extensive attention due to their unique advantages in making flexible and roll-to-roll solar cells via solution processed coating techniques ${ }^{1-5}$. The great progress has been made in the power conversion efficiencies (PCEs) thanks to the rapid development of nonfullerene acceptors (NFAs) ${ }^{6-11}$. In particular, the successful breakthroughs in Y6 series NFAs boosts the single junction $\mathrm{BHJ}$ OSCs efficiencies over $17-18 \%^{12-16}$. The $\mathrm{BHJ}$ strategy, however, has obvious intrinsic limitations for further improvement and technology deployment: (i) in laboratory spin coating optimization, the morphology evolution of $\mathrm{BHJ}$ photoactive layer during donor (D) and acceptor (A) mixture is complicated kinetically, which requires delicate balance of treatment conditions, such as D/A ratio, processing solvent and additive, thermal and/or solvent annealing ${ }^{17,18}$. The balance between the phase separation and phase purity remains extremely challenging for the sake of optimal charge separation and charge transport. The vertical components distributions remain uncertain in the present trial-and-error optimization, let alone to control with precision ${ }^{19}$. (ii) The BHJ performances depend strongly on the phase separation, which is closely related to the miscibility and solubility of donor and acceptor in the blend solution and thus limits the selection of processing solvents ${ }^{20,21}$. Major high-performance BHJ OSCs are processed using toxic solvents, such as chlorobenzene $(\mathrm{CB})$ and chloroform $(\mathrm{CF})^{22,23}$. The efficiencies of $\mathrm{BHJ}$ OSCs using nonhalogenated low-toxic solvents still lag behind the toxic solvent processed ones ${ }^{24}$; (iii) Due to the short diffusion length and amorphous properties of photovoltaic materials, charge transport pathways can be obstructed once thickening the $\mathrm{BHJ}$ film, which is originated from the increased trap densities in the D/A mixed regions with unfavorable phase separation close to the electrodes. Thus, fill factors (FFs) cannot be well maintained in the thick BHJ active layers, leading to the degradation of performances in thicker $\mathrm{OSCs}^{25,26}$. (iv) Moreover, when transferring the coating process to the blade coating, the $\mathrm{BHJ}$ morphology regulation becomes more complex ${ }^{27,28}$. Spin coating is a fast film drying process enabled by rapid airflow due to the centrifugal effect. During the blade coating process, the slow drying of donor and acceptor mixture wet film makes very different crystallization and intermixing dynamics, leading to totally different D-A phase separation morphologies and thus dramatic variation in the device performances $^{29-31}$.

Solar cell technology started as sharp $\mathrm{p}-\mathrm{n}$ junction structure in highly crystalline semiconductors. This structure does not work in low mobility and poor crystalline solar cell systems like amorphous silicon (a-Si), where $\mathrm{p}-\mathrm{i}-\mathrm{n}$ structure dominates. Polymeric OSC with revolutionary $\mathrm{BHJ}$ structure represents the other extreme of solar cell structures. The concept of gradient junction has also been proposed in solar cell field. After introducing wider bandgap a-SiC:H emitter for $\mathrm{p}-\mathrm{i}-\mathrm{n}$ heterojunction aSi solar cell, grading $\mathrm{p}$-i interface further advanced a-Si solar cell to $12 \%$ PCE. In GaAs solar cell, via varying $\mathrm{Al}$ fraction in AlxGa1-xAs, compositional grade in the $p$ layer can be achieved with GaAs near the pn junction, to a wider bandgap alloy at the front window layer surface. The gradient strategy can introduce electric field assisting electron migration, improve minority carrier collection and thus enhance the photocurrent ${ }^{32}$.

The historical technology progresses motivate us to take serious effort integrating controllable gradient concept with $\mathrm{BHJ}-$ forming graded $\mathrm{BHJ}(\mathrm{G}-\mathrm{BHJ})$ realized by sequential deposition (SD) method. In this method, we deposit the D and A layers sequentially, enabling optimization and regulation of the $\mathrm{D}$ and $\mathrm{A}$ individually with well-maintained crystallinity of materials ${ }^{33,34}$. Furthermore, the D layer and A layer are processed separately, leaving large freedom for the selection of processing solvents, especially considering the use of green solvents, e.g., toluene, $o$ xylene (XY) and tetrahydrofuran (THF), etc. towards highperformance OSCs ${ }^{34-36}$. SD is well utilized in the fullerene based OSCs to construct the comparable efficiencies to the BHJ OSCs, wherein dichloromethane (DCM)/1,2-Dichlorobenzene (o-DCB) solvents or DCM/CF are chosen as ideal orthogonal solvents to process fullerene derivatives and polymer donor layer, respectively, due to the distinct solubility of fullerenes and polymers in processing solvents. Consequently, with higher A concentration near the cathode and more $\mathrm{D}$ composition at the anode, the vertical graded $\mathrm{D}-\mathrm{A}$ distributions are more amenable to forming so-called $p-i-n$ structure ${ }^{37-39}$. On the other hand, since small molecular NFAs have similar backbones to polymer donors, the solubility in common solvents is similar, leading to the limited selection of orthogonal solvent as well as the insufficient D/A mixed interfaces formation ${ }^{40,41}$. Although high-performance NFAs-based OSCs via SD method have been previously reported $^{42-45}$, it lacks a clear and quantitative morphological picture. The absence of a rational guideline for solvent selection so far makes the fundamental mechanism underlying the G-BHJ film formation ambiguous. This work aims at removing the scientific ambiguity. When transferring the processing from laboratory spin coating to blade coating film-forming dynamics will be slow, and thus leads to the dramatically different active layer morphology with excessive aggregation of the printed active layer, especially for the NFAs, leading to the oversized domains in the blade coated active layer ${ }^{46}$. To solve this issue, great efforts, such as vacuum annealing, processing solvents engineering, novel material design etc., are made to facilitate the solidification towards reasonable scale of phase separation in high performance OSCs $27,31,47,48$. However, these optimization strategies are complicated and trial-and-error processes, and there are very few works reported in non-halogenated processed highly efficient blade-coated OSCs.

In this work, a simple yet effective G-BHJ formation strategy is demonstrated based on benchmark polymer PM6 donor and BTP-eC9 NFA OSC system. Optimal G-BHJ films from two solvents-XY and $\mathrm{CF}$ - are achieved and quantitatively studied by the depth-profiling X-ray photoemission spectroscopy (DP-XPS) technique. An indicative solvent guideline for achieving G-BHJ is provided based on tailored quasi-solid-state inter-diffusion of the two materials. Consequently, the optimal spin-coated G-BHJ OSCs processed by $\mathrm{CF}$ and XY deliver outstanding PCEs of $17.54 \%$ and $17.48 \%$, respectively. In particular, the XY-based GBHJ OSC affords a remarkable short-circuit current density $\left(J_{\mathrm{SC}}\right)$ of $26.65 \mathrm{~mA} \mathrm{~cm}^{-2}$ and an FF of 0.781 , which is one of highest binary OSCs from nonhalogenated solvent. Combined with angle-dependent grazing incidence X-ray diffraction (GI-XRD) measurements, gradient polymer composition and crystallinity distributions are quantitatively visualized: less polymer and weaker crystallinity at the cathode side, while more polymer and stronger crystallinity at the anode side, (and vice versa for acceptor). Noticeably, we found that the G-BHJ can maintain decent PCEs over 14\% when the thickness of the active layers is varied in a wide range from 120 to $500 \mathrm{~nm}$, which is rarely studied in the G-BHJ studies to the best of our knowledge. Thickness insensitive fabrication is much preferred in roll-to-roll printing. More importantly, nonhalogenated solvent enabled G-BHJ OSC via blade coating to achieve a record $16.77 \%$ PCE under open air condition, accompanied by an open-circuit voltage $\left(V_{\mathrm{OC}}\right)$ of $0.836 \mathrm{~V}$, a $J_{\mathrm{SC}}$ of $26.26 \mathrm{~mA} \mathrm{~cm}^{-2}$ and an FF of 0.764 . Furthermore, the excessive aggregation induced unfavorable phase separation due to the slow film drying in the conventional $\mathrm{BHJ}$ active layer was relieved in the blade-coated G-BHJ film, making G-BHJ strategy a feasible and promising strategy towards highly efficient, eco, and manufacture friendly OSCs. 
a

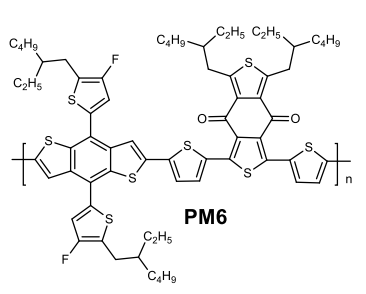

C
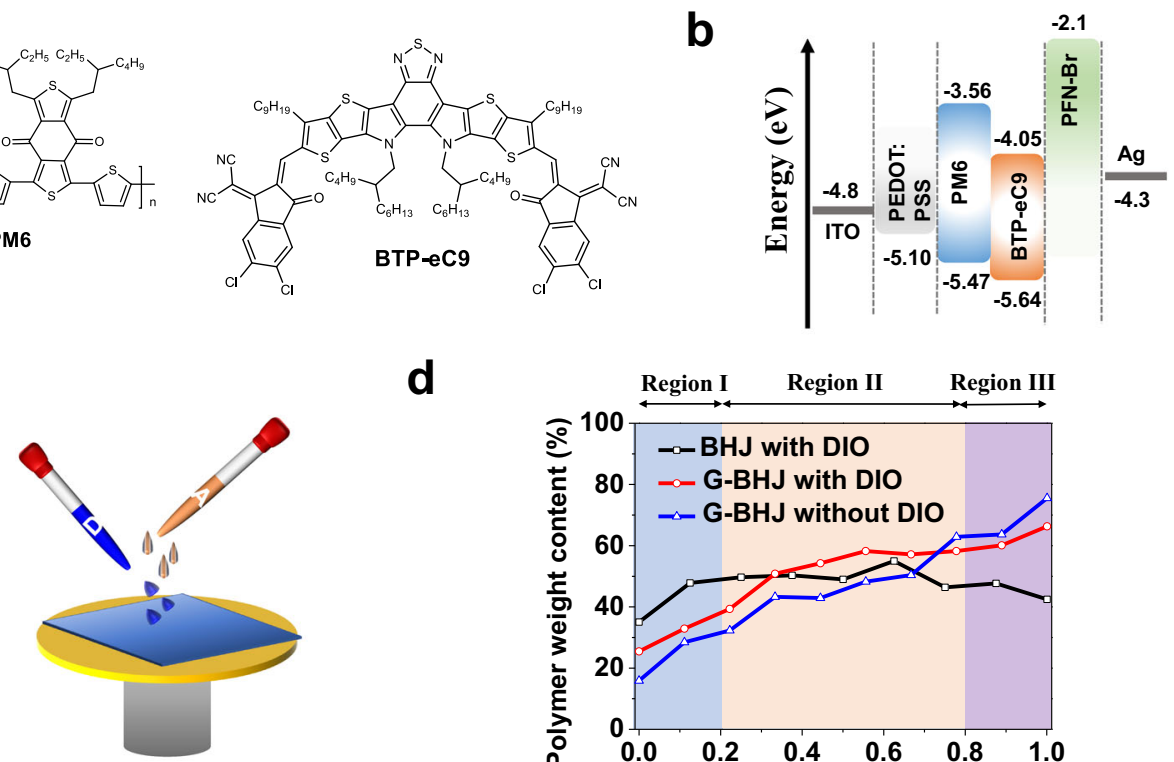

d

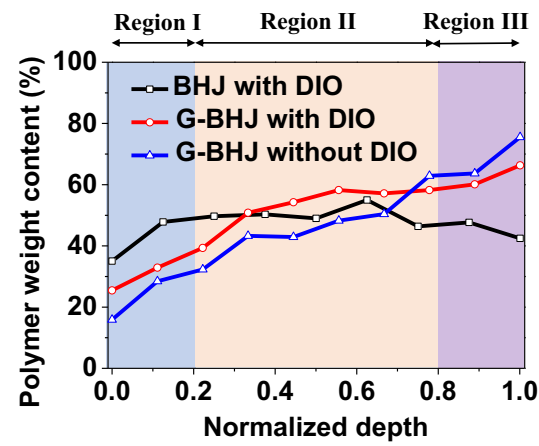

Fig. 1 Materials, optoelectronic properties and vertical composition distribution in BHJ and G-BHJ films. a Chemical structure of PM6 and BTP-eC9. b Schematic illustration of the conventional device structure of OSCs. c Schematic diagram of the sequential deposition spin coating procedure. $\mathbf{d}$ Variation of polymer weight content of BHJ with DIO, G-BHJ without DIO and G-BHJ with DIO films throughout the whole film.

\section{Results}

Solvent selection rules and G-BHJ formation. The chemical structures of the polymer donor PM6 and the small molecular acceptor (SMA) BTP-eC9 are shown in Fig. 1a. A conventional structure of indium tin oxide (ITO)/poly(3,4-ethylenedioxythiophene):polystyrene sulfonate (PEDOT:PSS)/active layer/ $\mathrm{PFN}-\mathrm{Br} / \mathrm{Ag}$ was adopted here and displayed in Fig. 1b. For sequential processing (Fig. 1c), the solution of neat donor PM6 was firstly deposited on the PEDOT:PSS layer, followed by depositing the neat acceptor solution on top. The solvent properties of upper layer are essential to morphology of the whole blend $^{49}$. Solvent properties including volatile/evaporation rate (boiling point) and solubility (acceptor and donor) need to be taken into consideration to construct successful SD films. Firstly, we varied the processing solvents of upper layer to fabricate $\mathrm{SD}$ films: THF, XY, CF, and CB with boiling points of $66^{\circ} \mathrm{C}, 140^{\circ} \mathrm{C}$, $62{ }^{\circ} \mathrm{C}$ and $132^{\circ} \mathrm{C}$, respectively. As shown in the optical images in Supplementary Fig. 1, SD-films processed by CF and XY were visually uniform and smooth, while the SD-film from THF solution was grainy and rough. Pinholes are clearly seen in the SD-film processed by $\mathrm{CB}$ due to the destruction of PM6 layer. In terms of solubility, $\mathrm{CB}$ and $\mathrm{CF}$ can easily dissolve SMA at room temperature, while SMA is found to be poorly soluble in THF and $\mathrm{XY}$ at room temperature, and thus high temperatures $\left(60^{\circ} \mathrm{C}\right.$ and $80^{\circ} \mathrm{C}$ ) are needed to dissolve it. Overall, solvent with poor solubility but fast evaporation like THF makes it hardly to achieve a high-quality SD-film. Similarly, solvent with excellent solubility and slow evaporation rate like $\mathrm{CB}$ cannot enable high-quality SDfilm neither. To further confirm the diffusion of acceptor into donor layer, the donor layer PM6 was washed by same amount of $\mathrm{XY}$ solvent, and the GI-XRD technique was used to study the film. Although weaker, the scattering signals maintained characteristic scattering peaks (Supplementary Fig. 2) well, indicative of the XY solvent wash does not disturb the microstructure of PM6 much.

Aiming at elucidating the mechanism, we conducted DP-XPS measurements to quantitatively investigate vertical composition distributions by detecting the atomic ratios throughout the whole
SD films ${ }^{37}$. All the samples were prepared on ITO/PEDOT:PSS substrates, as the same as the real devices. Since both PM6 and BTP-eC9 contain oxygen $(\mathrm{O})$, while only PM6 contains fluorine (F), we attribute the O element to both PM6 and BTP-eC9, F atoms to only PM6. One repeat unit of PM6 contains two F atoms and two $\mathrm{O}$ atoms, and one BTP-eC9 molecule contains two $\mathrm{O}$ atoms. Based on the $\mathrm{O} / \mathrm{F}$ atom ratio, the polymer weight content (wt\%) at different depths can be calculated (see more details in Supplementary Table 1 and Supplementary Note 1$)^{50}$. As shown in Fig. 1d, the conventional BHJ film with DIO displayed a small portion of donor polymer $(\sim 45 \mathrm{wt} \%)$ at the bottom region, which are generally considered to be negative for charge extraction at the anode. Overall, both SD films with and without DIO treatment display a clear graded polymer distribution from the top surface to the bottom: BTP-eC9-enriched at the top, and PM6-enriched at the bottom, which is clearly distinguished from the BHJ film's vertical polymer distribution. This proves that the SD method creates a graded BHJ-like morphology, conceptually proposed as "G-BHJ" here.

In the G-BHJ films deposited with and without DIO additive, in addition to the BTP-eC9-enriched at the top (region I), PM6enriched at the bottom (region III), interestingly there is a bulk region with less variation (region II) in between. For the G-BHJ without DIO, a pronounced gradient composition distribution of polymer from the top region I ( $15.8 \mathrm{wt} \%)$, to the bottom region III (75.5 wt\%) was observed. The concentrated PM6 at region III suggests that the weaker interdiffusion can proceed to the deeper region of PM6 layer. While after adding DIO, more polymer contents are located at the bulk region II accompanied by less polymer content ( $66.4 \mathrm{wt} \%)$ enriched at region III, implying that the DIO additive could assist the downshift movement of BTPeC9 acceptor molecules into PM6 domains. This represents a new morphology manipulation approach compared to those in traditional BHJ. Using the same DP-XPS technique, for SDfilms processed by chlorinated solvent $(\mathrm{CF})$, the results verified its graded vertical composition distribution derived from the increasing polymer weight vertically from region I to region III (see Supplementary Fig. 3 and Supplementary Table 2), which 
matches with the previous CF-based SD studies ${ }^{51}$. To further confirm G-BHJ morphology, time-of-flight secondary-ion mass spectrometry (ToF-SIMS) profiling technique was applied to monitor the vertical profile of donor and acceptor by taking XYbased G-BHJ film (with DIO) as an example (on PEDOT:PSS too, Supplementary Fig. 4). The signal of $\mathrm{CN}^{-}$showed that the BTPeC9 contents were obviously enriched at the top region, and then decreased gradually to the middle bulk region. The PM6 concentration shows an obvious plateau in the middle part followed by a continuously increasing trend at the bottom region. These results are well consistent to the DP-XPS results wherein G-BHJ morphology is solidly shaped.

The results above provide solid evidence of two successful yet distinct pathways towards valid G-BHJ morphology: (1) high boiling point solvent features longer time for interdiffusion between $\mathrm{A}$ and $\mathrm{D}$, yet limited solubility indicates smaller penetration rate of $\mathrm{A}$, leading to proper degree of A's interdiffusion into D domain (XY-case); (2) low boiling point solvent enables shorter time for A to penetrate downward, yet good solubility provides larger penetration rate of $\mathrm{A}$, reaching similarly functional interdiffusion of A into D domain (CF-case). Hence, the vertical composition profiles of $\mathrm{A}$ and $\mathrm{D}$ can be finely regulated by the penetration rate and time. Overall, by delicately tailoring the balance of solubility and boiling point of upper solvent, G-BHJ would be expected to be formed. Additive engineering provides a further dimension of morphology manipulation as mentioned before. This offers a simple guideline of solvent selection rule for rational G-BHJ morphology formation by tuning the interdiffusion between $\mathrm{A}$ and $\mathrm{D}$.

Device performance. Based on this G-BHJ concept, we fabricate the OSCs to evaluate the photovoltaic performances for both traditional BHJ and G-BHJ OSCs. For the XY-processed G-BHJ OSCs, the absorption profile was displayed in Supplementary Fig. 5. Optimizing the thickness of donor and the acceptor layer was done by changing spin speed and the 1,8-diiodooctane (DIO) additive amount (See Supplementary Fig. 6 and Supplementary Tables 3-5). With the optimized D/A thickness of 70/55 nm and $0.5 \%$ DIO, the best-performing G-BHJ device delivered maximum PCE of $17.48 \%$ with a short-circuit current density $\left(J_{\mathrm{SC}}\right)$ of $26.65 \mathrm{~mA} \mathrm{~cm}^{-2}$, an open-circuit voltage $\left(V_{\mathrm{OC}}\right)$ of $0.840 \mathrm{~V}$, and an $\mathrm{FF}$ of 0.781 . The fabrication of $\mathrm{BHJ}$ was provided in Supplementary Methods. The BHJ reference device, as shown in Table 1, afforded an optimized PCE of $16.41 \%$ with a $J_{\mathrm{SC}}$ of $25.75 \mathrm{~mA} \mathrm{~cm}^{-2}$ and an FF of 0.760 . As expected, the optimal G-BHJ device from CF solution exhibited a higher PCE of $17.54 \%$ in comparison to BHJ with a PCE of $17.08 \%$ (see Supplementary Fig. 7 and Supplementary Table 6). We also study the performances of THFbased and CB-based SD devices, as well as their BHJ counterparts as a comparison (Supplementary Fig. 8 and Supplementary Table 7). The inferior performances of THF-based and CB-based
SD devices compared to their $\mathrm{BHJ}$ counterparts are due to the failure of constructing effective G-BHJs. For comparison, the BHJ devices made from CB (17.58\%), CF (17.08\%), and XY (16.41\%) all give relatively good performance, while THF completely fails in fabricating $\mathrm{BHJ}$ device due to solubility issue, especially in donor polymer. The fact that SD device processed by THF still enables 9.68\% PCE further indicates the bigger freedom in constructing OSCs. With the distinct advantage of nonhalogenated solvent processed G-BHJ OSC in the future practical application, we focus on $\mathrm{XY}$ processed G-BHJ OSCs from now on.

The current density-voltage $(J-V)$ characteristics of the optimal devices and corresponding performance parameters are depicted in Fig. 2a and Table 1. The PCEs of G-BHJ OSCs outperform the corresponding $\mathrm{BHJ}$ control device, with synergistically enhanced $V_{\mathrm{OC}}, J_{\mathrm{SC}}$, and FF. It is worth-noting that the trade-off between the $J_{\mathrm{SC}}$-FF was found to be effectively alleviated in the G-BHJ film. Furthermore, the efficiencies processed by nonhalogenated solvent is approaching to that processed from CF solvents in the previous reports ${ }^{13}$. The external quantum efficiency (EQE) of the optimal BHJ and G-BHJ based devices were displayed in Fig. $2 \mathrm{~b}$. The integrated $J_{\mathrm{SC}}$ values of $\mathrm{BHJ}$ and G-BHJ OSCs calculated from the EQE curves are $25.30 \mathrm{~mA} \mathrm{~cm}^{-2}$ and $26.04 \mathrm{~mA} \mathrm{~cm}^{-2}$, respectively, as listed in Table 1 , which are consistent to those obtained from the $J-V$ curves (within $2.5 \%$ error). Compared with the BHJ devices, the EQE values of G-BHJ devices were enhanced, mainly due to the improvement in the wavelength region corresponding to the PM6 and BTP-eC9 absorption with the highest EQE of $88 \%$ at $\sim 650 \mathrm{~nm}$, which can be clearly seen from the EQE differences between $\mathrm{BHJ}$ devices and G-BHJ devices. Figure $2 c$ demonstrates the efficiency histograms of $\mathrm{BHJ}$ and $\mathrm{G}-\mathrm{BHJ}$ OSCs.

Carrier dynamics analysis. The improved $J_{\mathrm{SC}}$ and $\mathrm{FF}$ are the main factors that contribute to the enhanced PCEs of G-BHJ OSCs. To better understand the exciton dissociation and charge collection mechanism in the active layers, the photocurrent density $\left(J_{\mathrm{ph}}\right)$ as a function of effective voltage $\left(V_{\text {eff }}\right)$ was plotted for the optimal BHJ and G-BHJ OSCs, as plotted in Supplementary Fig. 9. Here, $J_{\mathrm{ph}}=J_{\mathrm{L}}-J_{\mathrm{D}}$, where $J_{\mathrm{L}}$ and $J_{\mathrm{D}}$ are the current densities under illumination and in the dark, respectively. $V_{\text {eff }}=V_{0}-V_{\mathrm{a}}$, where $V_{0}$ is the voltage when $J_{\mathrm{L}}$ equals $J_{\mathrm{D}}$ and $V_{\mathrm{a}}$ is the applied voltage. On the basis of this curve, the ratio of $J_{\mathrm{ph}} / J_{\mathrm{sat}}$ (the saturation $J_{\mathrm{ph}}$ ) under the short-circuit condition relates to exciton dissociation efficiency $\left(\eta_{\text {diss }}\right)$, and the charge collection efficiency $\left(\eta_{\text {coll }}\right)$ is evaluated by the ratio of $J_{\mathrm{ph}} / J_{\mathrm{sat}}$ at the maximal power output point. The related parameters above are provided in Supplementary Table 8 . The $\eta_{\text {diss }}$ value of the optimized G-BHJ devices is $96.03 \%$, which is slightly larger than that of the $\mathrm{BHJ}$ devices. The improved $\eta_{\text {diss }}$ and $\eta_{\text {coll }}$ for the optimized G$\mathrm{BHJ}$ device demonstrates a better exciton dissociation and charge

Table 1 Device performances of BHJ and G-BHJ OSCs via spin coating and blade coating processes under the illumination of an AM 1.5 G solar simulator, $100 \mathrm{~mW} \mathrm{~cm}^{-2}$.

\begin{tabular}{|c|c|c|c|c|c|}
\hline & $V_{\text {oc }}(\mathbf{V})$ & $J_{\mathrm{SC}}(\mathrm{mA} \mathrm{cm}-2)$ & $\mathbf{F F}$ & $\mathrm{PCE}_{\max }\left(\mathrm{PCE}_{\mathrm{avg}}\right)^{\mathrm{a}}(\%)$ & $J_{\text {calc. }}{ }^{b}$ \\
\hline $\mathrm{BHJC}$ & $0.839(0.838 \pm 0.002)$ & $25.75(25.59 \pm 0.24)$ & $0.760(0.755 \pm 0.010)$ & $16.41(16.20 \pm 0.11)$ & 25.30 \\
\hline $\mathrm{BHJ}$ & $0.835(0.835 \pm 0.003)$ & $25.24(25.27 \pm 0.15)$ & $0.753(0.747 \pm 0.005)$ & $15.87(15.78 \pm 0.06)$ & 24.92 \\
\hline $\mathrm{G}-\mathrm{BHJC}$ & $0.840(0.838 \pm 0.002)$ & $26.65(26.55 \pm 0.42)$ & $0.781(0.772 \pm 0.008)$ & $17.48(17.19 \pm 0.20)$ & 26.04 \\
\hline
\end{tabular}

The errors are defined as standard deviation.

aAverage PCE from ten independent cells.

bdenotes integrated $J_{\text {sc }}$ from the EQE curves.

CThe optimal OSCs via spin coating.

${ }^{\mathrm{d}}$ The optimal OSCs via blade coating 
a

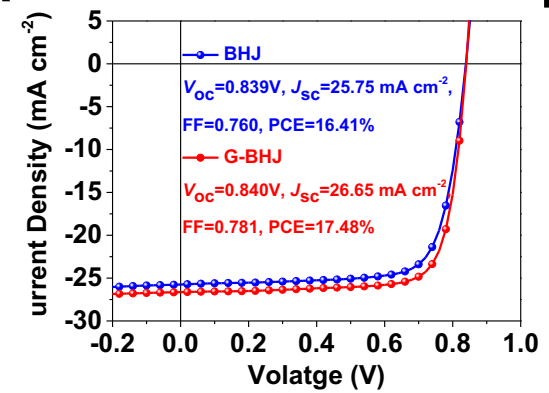

C

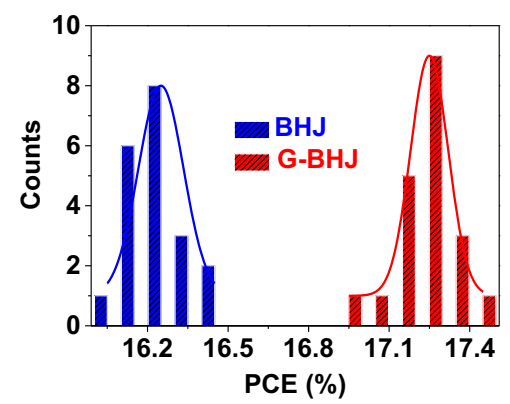

b

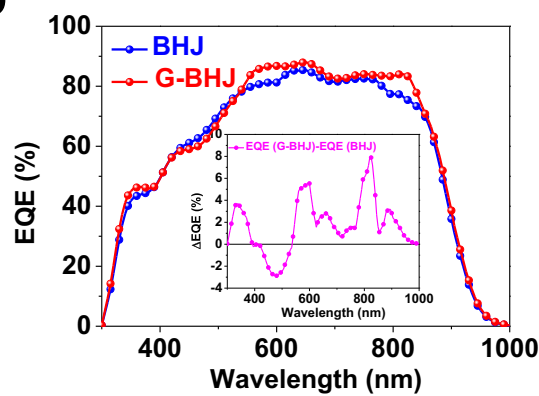

d

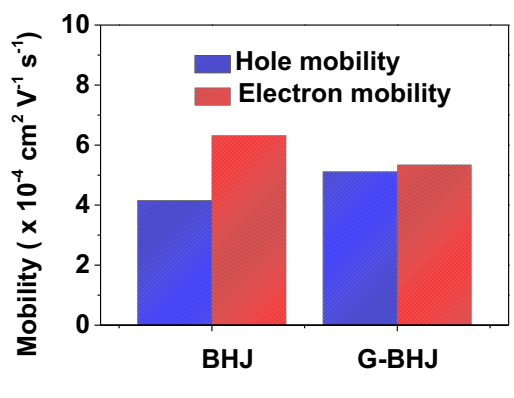

Fig. 2 Device performances. a $J-V$ curves and $\mathbf{b}$ EQE response of best-performing BHJ and G-BHJ-based devices. c Histograms of the PCE counts for 20 optimal BHJ- and 20 optimal G-BHJ-based devices. d Histogram of hole and electron mobilities for BHJ and G-BHJ blends determined by SCLC measurements.

collection, well explain the synchronous enhancement of $J_{\mathrm{SC}}$ and FF in the G-BHJ OSCs.

In addition, the slope of $n k T / q$ in the function of $V_{\mathrm{OC}} \propto$ $n k T / q \ln P$ ( $k$ is Boltzmann constant, $q$ is elementary charge, and $T$ is temperature) is an indicator of the trap-assisted recombination (monomolecular recombination), which could be extracted by plotting $V_{\mathrm{OC}}$ versus the natural logarithm of $P$ (Supplementary Fig. 10a). The comparable fitted slopes between BHJ (1.24kT/q) and G-BHJ devices $(1.21 \mathrm{kT} / \mathrm{q})$ indicate that the G-BHJ strategy shows similar trap-assisted charge recombination.

With regard to the bimolecular recombination loss, the dependence of $J_{\mathrm{SC}}$ on the $P$ can be described by the power law relationship $\left(J_{\mathrm{SC}} \propto P^{\mathrm{s}}\right)$, where $S$ is the exponential factor related to bimolecular recombination. As shown in Supplementary Fig. 10b, the calculated $S$ value for the optimal G-BHJ device is approaching unity (0.98), larger than that of $\mathrm{BHJ}$ device (0.95), reflecting the weak bimolecular recombination loss under short circuit condition. The reduced charge recombination losses (monomolecular and bimolecular recombination) are assumed to benefit from the expected vertical phase separation with acceptor enriched at the cathode and donor enriched at the anode sides.

The photoluminescence (PL) quenching efficiency was employed to further investigate the charge dissociation properties in different films. As displayed in Supplementary Fig. 11, the neat PM6 and BTP-eC9 exhibited distinct PL spectra with maximal emission peaks located at $682 \mathrm{~nm}$ and $936 \mathrm{~nm}$, respectively. Both the $\mathrm{BHJ}$ and G-BHJ films exhibit efficient PL quenching. The PL intensities of $\mathrm{BHJ}$ and G-BHJ films decrease by $97.6 \%$ and $99 \%$, respectively, compared to that of the neat PM6 film (Supplementary Fig. 11a). Similarly, the quenching efficiencies of $\mathrm{BHJ}$ and $\mathrm{G}-\mathrm{BHJ}$ films, relative to the neat $\mathrm{BTP}-\mathrm{eC}$, are calculated to be $94.5 \%$ and $98.1 \%$, respectively (Supplementary Fig. 11b). These positive results suggest that the quenching efficiencies of $\mathrm{G}-\mathrm{BHJ}$ film is even stronger than that of $\mathrm{BHJ}$ film, implying that the sufficient D/A interfaces are well formed for efficient charge transfer in the G-BHJ OSCs using non-orthogonal solvents, contributing to the higher $J_{\mathrm{SC}}\left(26.65 \mathrm{~mA} \mathrm{~cm}^{-2}\right)$.
To explore the improvement of FF in G-BHJ based devices, we investigated the charge transport properties in the $\mathrm{BHJ}$ and G$\mathrm{BHJ}$ based devices by fabricating the electron-only diodes using the structure of ITO/ZnO/BHJ (G-BHJ) active layer/Ag and holeonly diodes using the architectures of ITO/PEDOT:PSS/ BHJ (G$\mathrm{BHJ}$ ) active layer/Au, respectively. By fitting the $J-V$ curves in the space-charge-limited current region (SCLC) in Supplementary Fig. 12. The detailed hole mobilities $\left(\mu_{\mathrm{h}}\right)$ and electron mobilities $\left(\mu_{\mathrm{e}}\right)$ values are calculated and summarized in Supplementary Table 9. The $\mu_{\mathrm{e}}$ of the optimal BHJ and G-BHJ devices are calculated to be $6.32 \times 10^{-4}$ and $5.34 \times 10^{-4} \mathrm{~cm}^{2} \mathrm{~V}^{-1} \mathrm{~s}^{-1}$, while the $\mu_{\mathrm{h}}$ of them are $4.15 \times 10^{-4}$ and $5.11 \times 10^{-4} \mathrm{~cm}^{2} \mathrm{~V}^{-1} \mathrm{~s}^{-1}$, respectively (Fig. $2 \mathrm{~d}$ ). There are two main factors affecting the charge transport: molecular packing and transportation pathways ${ }^{50}$. The reason of reduced $\mu_{\mathrm{e}}$ and the improved $\mu_{\mathrm{h}}$ in the optimal G-BHJ devices may be due to the strengthening of the polymer crystallinity and reduced crystallization of acceptor, which will be discussed in the GIWAXS part. As a result, a more balanced $\mu_{\mathrm{e}} / \mu_{\mathrm{h}}$ of 1.05 was achieved for the G-BHJ devices in comparison with that of 1.52 in the $\mathrm{BHJ}$ counterparts, indicative of more balanced charge transport properties and less isolated domains in the optimal G-BHJ devices, leading to less charge recombination and thus higher $\mathrm{FF}(\approx 0.78)$ can be achieved.

Molecular packing and crystallinity analysis. To further understand the vertical morphology characteristics in the $\mathrm{BHJ}$ and G-BHJ active layers, the GI-XRD measurements were performed, giving details of the molecular packing and crystallinity. To give the vertical crystallinity information across the whole film, angle-dependent GI-XRD was performed in this study. The functional relationship between theoretical penetration depth of $\mathrm{X}$-ray beam and incident angle was depicted in Supplementary Fig. 13. If the incident angle of X-ray $(\alpha)$ is smaller than critical angel $\left(\alpha<\alpha_{\mathrm{c}}=\sim 0.2^{\circ}\right)$, the top $\sim 7 \mathrm{~nm}$ (upper layer) of the film can be detected. If $\alpha>\alpha_{c}$, the whole depth of film can be penetrated, from which we can map the overall crystallinity of the film. The 

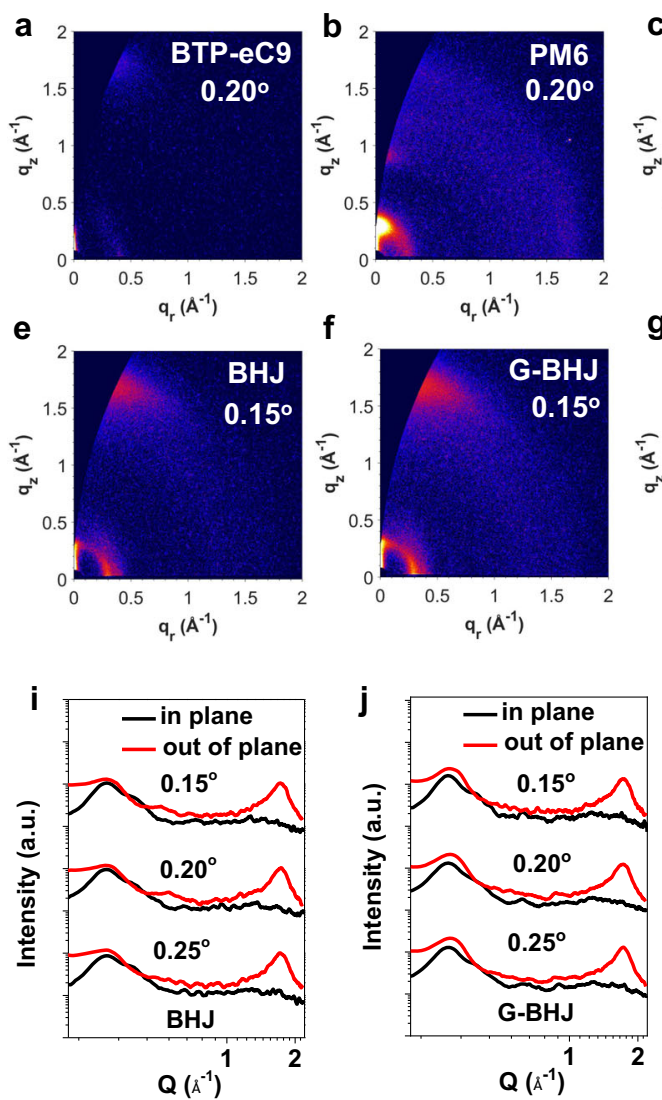
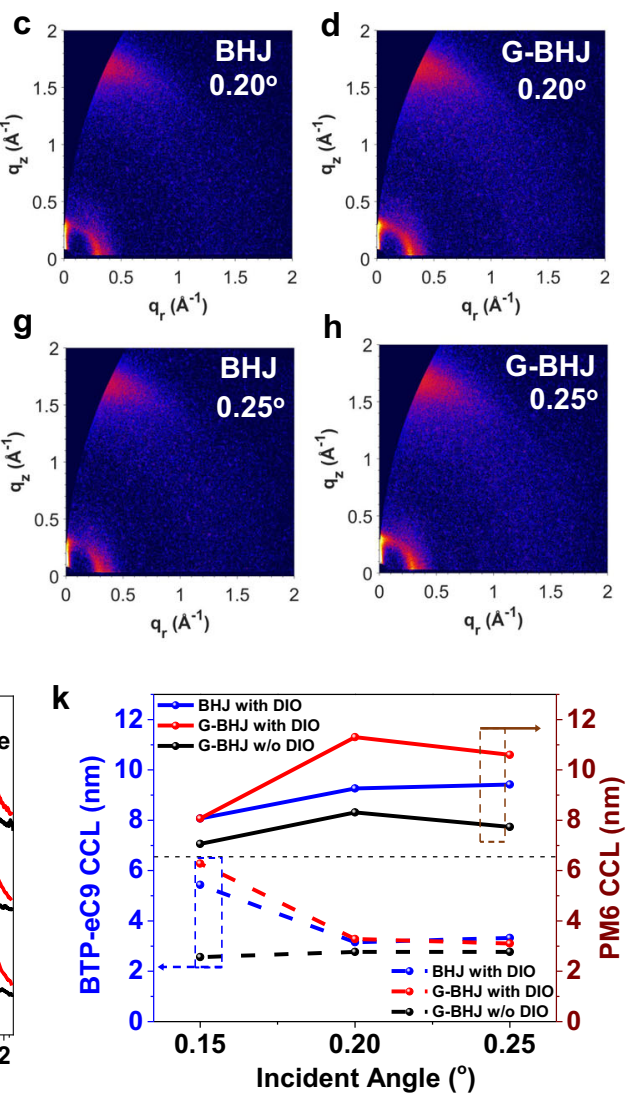

Fig. 3 Molecular packing and crystallinity of BHJ and G-BHJ films. 2D GIWAXS patterns of $\mathbf{a}$ neat BTP-eC9 film, $\mathbf{b}$ neat PM6 film, c the optimal BHJ and d the optimal G-BHJ processed films at critical incident angle of $0.20^{\circ}$. 2D GIWAXS patterns of $\mathbf{e}$ the optimal $\mathrm{BHJ}$ and $\mathbf{f}$ the optimal G-BHJ films collected at incident angles of $0.15^{\circ}$. 2D GIWAXS patterns of $\mathbf{g}$ the optimal $\mathrm{BHJ}$ and $\mathbf{h}$ the optimal $\mathrm{G}$ - $\mathrm{BHJ}$ films collected at incident angles of $0.25^{\circ}$. The corresponding 1D linecut profiles of $\mathbf{i}$ the optimal $\mathrm{BHJ}$ and $\mathbf{j}$ the optimal $\mathrm{G}-\mathrm{BHJ}$ films at three different $\mathrm{X}$-ray incident angles of $0.15^{\circ}, 0.20^{\circ}$ and $0.25^{\circ}$, respectively. k Coherence lengths of PM6 and BTP-eC9 in three films versus varied incident angles.

two-dimensional (2D) GI-XRD patterns are shown in Fig. 3a-h and the corresponding line profiles in the in-plane (IP) and outof-plane (OOP) directions are extracted in Fig. 3i, j. Firstly, critical incident angle of about $0.2^{\circ}$ was chosen to reveal the average crystallinity of the neat materials as well as the optimal $\mathrm{BHJ}$ and $\mathrm{G}-\mathrm{BHJ}$ active layers throughout the whole film ${ }^{52}$. The neat BTPeC9 displayed the preferred face-on orientation with a clear $\pi-\pi$ stacking peak at $q \sim 1.76 \AA^{-1}$ in the OOP direction and a lamellar peak at $q \sim 0.40 \AA^{-1}$ in the IP direction, which is consistent with the previous reports ${ }^{13}$. The pristine PM6 also adopts the face-on orientation with respect to the substrate, showing a lamellar peak at $q \sim 0.30 \AA^{-1}$ along the IP direction and a $\pi-\pi$ stacking peak (010) at $q \sim 1.69 \AA^{-1}$ along the OOP direction, respectively. For the blend films, it was found that both BHJ film and G-BHJ film maintained the face-on orientation, indicating the G-BHJ approach does not change the molecular orientation property. Noticeably, both BHJ and G-BHJ films showed two obvious characteristic peaks in the IP direction at lower $q$ region, which can be assigned to the PM6 with higher intensity at $q \sim 0.3 \AA^{-1}$ and BTP-eC9 with lower intensity at $q \sim 0.4 \AA^{-1}$, respectively. Thanks to this, peak fitting analysis was conducted to reveal the different impacts of $\mathrm{BHJ}$ and G-BHJ methods on the packing ordering of individual components. This was done by taking line cuts from the GI-XRD patterns in the IP direction and assigning the IP peaks to individual components. Crystalline coherence lengths (CCL) can be used to estimate the crystal size of the domains, which could be determined using Scherrer equation: $\mathrm{CCL}=2 \pi K / \mathrm{FWHM}$, where $K$ is a shape factor (here is 0.9 ) and FWHM is the full width at half-maximum of each peak ${ }^{53}$. The detailed lamellar stacking CCL and $\pi-\pi$ stacking CCL data are summarized in the Supporting Information. The calculated CCL of PM6 lamellar stacking $(100)\left(q \sim 0.29 \AA^{-1}\right)$ was $92.6 \AA$ in the BHJ film, while the CCL for the G-BHJ film increased to $113 \AA$, meanwhile the d-spacing of (100) packing decreased (Supplementary Table 10), indicating the ordering of packing for PM6 was enhanced in the G-BHJ film. For the small molecular NFA, it was found that both $\mathrm{BHJ}$ and G-BHJ films can induce the lamellar diffraction from BTP-eC9 with similar CCLs (Supplementary Table 10). Furthermore, both BHJ and G-BHJ films possess strong $\pi-\pi$ stacking peaks of (010) along the OOP direction, which should belong to the combination of PM6 and BTP-eC9 and difficult to distinguish. The calculated (010) CCLs of BHJ and G-BHJ films are estimated to be $20.6 \AA$ and $19.9 \AA$ (Supplementary Table 11), respectively, showing similar $\pi-\pi$ stacking structure. Thus, utilizing non-orthogonal solvent driven G-BHJ approach is demonstrated to facilitate the crystallization structure of PM6, which can be explained by the fact that PM6 is solidified first to form a scaffold and could maintain the pristine nanostructure to certain degree in the XY solvent during the acceptor coating. The enhanced crystallinity of polymer is in excellent agreement with the improved hole mobility as discussed above.

The smaller grazing angle of $0.15^{\circ}$ was chosen to detect the crystallization properties at the upper layer $(\sim 7 \mathrm{~nm})$. The upper layer GI-XRD results revealed the BTP-eC9 IP lamellar stacking peaks (100) existing in both $\mathrm{BHJ}$ and G-BHJ films, indicating the existence of BTP-eC9 crystallites on the top layer. Further peak analysis revealed that the optimal G-BHJ film processed larger 
lamellar stacking CCL ( 62.8 $)$ for BTP-eC9 than that of BHJ film ( 54.3 $\AA$ ) (Supplementary Table 10), confirming more ordered BTP-eC9 aggregates formation at the surface of G-BHJ films and thus providing more continuous pathways for electron transport. The crystal sizes of PM6 donor at the top layer, on the other hand, are almost the same. We also chose larger incident angle larger than critical angle $\left(0.25^{\circ}\right)$ to detect the average crystalline structure of the entire film. The CCL of PM6 lamellar stacking increased significantly from the incident angle of $0.15^{\circ}$ to $0.25^{\circ}$-from $80.7 \AA \mathrm{nm}$ to $102 \AA$ in the G-BHJ film and $80.7 \AA$ to $94.2 \AA$ in the BHJ film (Supplementary Table 10), respectively, indicating larger gradient of PM6 crystalline nanostructure in the G-BHJ film from the top surface to the substrate. On the contrary, the crystal size of BTP-eC9 displayed a decreased trend from the top surface to the bottom substrate, giving the evidence that the aggregation of small molecules can be inhibited when the small molecules diffuse towards the bottom layer. This is reasonable because the interdiffusion processes and crystal nucleuses growth of BTP-eC9 are competitive dynamic processes ${ }^{42}$. Also, the enhanced crystallization of PM6 at the bottom potentially could further restrict the self-assembly of BTP-eC9. Generally, the (010) $\pi-\pi$ stacking show the similar trend with lamellar stacking (Supplementary Table 11). The more ordered BTP-eC9 structure on the top and improved crystallinity of PM6 on the bottom in the optimal G-BHJ active layer contributes to the gradient vertical crystallinity towards more valid charge transport properties, leading to the higher $J_{\mathrm{SC}}$ and FF. Furthermore, we also measured the angle-dependent GI-XRD of the G-BHJ film without additive to elucidate the functional role of DIO on the vertical crystallinity in the G-BHJ film (Supplementary Fig. 14 and Supplementary Tables 10,11 ), which is rarely studied in the sequential deposited OSCs. The simultaneously prolonged CCLs for the lamellar stacking of PM6 and BTP-C9 throughout the whole film after the incorporation of DIO, especially the BTP-C9 on the top and PM6 on the bottom, demonstrate that $0.5 \%$ DIO could induce the strong growth of crystallites for both polymer and small molecules. Furthermore, the more balanced crystallization between the polymer donor and small molecular acceptor from top to bottom can be clearly observed, which indicates that DIOassisted G-BHJ strategy could finely reduce the crystallization gap of $\mathrm{D}-\mathrm{A}$ domain. To assist the visualization, the vertical evolution of crystal size (CCL) for respective PM6 and BTP-eC9 is presented in Fig. 3k. The synergetic effect of G-BHJ (by SD process) and delicately tuned/enhanced D-A crystallinity (by additive) is behind the success of optimal G-BHJ devices.

Atomic force microscope (AFM) and transmission electron microscopy (TEM) characterizations were further employed to study the surface and bulk morphologies of blend films. As shown in Fig. 4a-c, AFM images of three films exhibit apparently different phase separation features on the top layer. For example, in the phase images (Fig. 4d-f), the fibril-like aggregation can be clearly observed in the BHJ blend film, which is closely dependent on the crystalline feature of polymer donor PM6, while the surface morphology of the G-BHJ film without DIO tends to form uniformly distributed granular aggregates, which is assumed to be due to the strong crystallization of BTP-eC9. Particularly, after DIO addition, the G-BHJ film shows the possibly more optimal phase separation with appropriate domain size, that features both polymer threadiness and small molecular granule, implying that the DIO additive could assist the upshift movement of PM6 for realizing sufficient intermixed regions. The mean-square surface roughness $\left(R_{\mathrm{q}}\right)$ of optimal $\mathrm{BHJ}$ film, G-BHJ film without DIO and optimal G-BHJ film are found to be $1.68 \mathrm{~nm}, 0.91 \mathrm{~nm}$ and $1.62 \mathrm{~nm}$, respectively. The AFM results are well consistent with the GIWAXS measurements. The TEM images of the three films exhibit similarity, indicating similar overall D-A phase-separation morphologies in the bulk part (See Supplementary Fig. 15), as TEM gives information of the whole film.

Based on the AFM (on surface) and TEM (on bulk) observations, we infer that the intermixed D/A microstructure is successfully evolved due to the easy swelling of PM6 material in $\mathrm{XY}$ when depositing the acceptor. However, the more pronounced vertical phase separations tuned by the G-BHJ method is expected, as evidenced vide supra, which brings about simultaneous gain of charge separation and transport and thus effectively reduce the trade-off between $J_{\mathrm{SC}}$ and FF. Finally, combined with DP-XPS and morphology results, we capture the picture of the vertical evolution morphology in BHJ and G-BHJ photoactive layers, respectively, as shown in Fig. 4g, h. In G-BHJ, less polymer with weaker crystallinity at the cathode side, while more polymer with stronger crystallinity at the anode side, (and vice versa for acceptor concentration and crystallinity distribution).

Thick-film G-BHJ OSCs. Inspired by the advantages of more balanced hole and electron mobilities and superior vertical morphological structure in the G-BHJ approach, we explored thick-film OSCs (up to $500 \mathrm{~nm}$ ) by varying the concentration of donor and acceptor and their spin coating speeds. Noticeably, the thickness effects of donor and acceptor on the photovoltaic performances are rarely studied in the previous sequential deposition literature. The $J-V$ curves G-BHJ OSCs are displayed in Fig. 5a and the corresponding photovoltaic parameters are summarized in Table 2. Thick-film based BHJ OSCs were also fabricated as comparison and the detailed results are provided in Supplementary Methods. As shown in Fig. 5b, broader and higher EQE spectra are observed for thick OSCs, the integrated $J_{\mathrm{SC}}$ from $\mathrm{EQE}$ spectra are well consistent with the $J_{\mathrm{sc}}$ measured in $J-V$ curves within small error (less than 3.5\%). As illustrated in Fig. $5 c, V_{\mathrm{OC}}$ values slightly decreased with the increase of thickness from $120 \mathrm{~nm}$ to $500 \mathrm{~nm}$, while $J_{\mathrm{SC}}$ values increase from 26.65 to $27.42 \mathrm{~mA} \mathrm{~cm}^{-2}$ when the thickness increased from 120 to $400 \mathrm{~nm}$ due to the enhanced photon absorption. Although FF has a reduction to some extent for devices thicker than typical $120 \mathrm{~nm}$, it remained the high values of 0.728 for $300 \mathrm{~nm}$ and 0.670 for $400 \mathrm{~nm}$, which are outstanding in the previous reported thickfilm based OSCs in both binary and ternary systems (Supplementary Table 12). The decrease of FF is partially related to the low mobility of organic photovoltaic materials, leading to the serious bimolecular recombination in the thick active layer ${ }^{12}$. It was worth-noting that G-BHJ OSCs possess higher tolerance to the wide range of thickness up to $500 \mathrm{~nm}$ compared to the $\mathrm{BHJ}$ OSCs (Supplementary Fig. 16 and Supplementary Table 13), mainly resulting from the superior FFs. For $\mathrm{BHJ}$ devices, the FF drops from 0.76 in $110 \mathrm{~nm}$ to 0.58 in $500 \mathrm{~nm}$ OSC, while in G$\mathrm{BHJ}$, the drop is much milder-from 0.78 in $120 \mathrm{~nm}$ to 0.64 in $500 \mathrm{~nm}$. The well maintained FFs in thick OSCs is also a clear indication that the system has smaller non-geminate recombination in G-BHJ devices. Finally, high efficiencies of $16.25 \%$ for $300 \mathrm{~nm}$ and $15.12 \%$ for $400 \mathrm{~nm}$ active layer are realized for GBHJ OSCs, respectively, which are among the highest values for OSCs with thick active layer (Fig. 5d), including both the binary and ternary OSCs ${ }^{12,54-67}$. To further support the G-BHJ morphology in thick device, DP-XPS characterization was conducted for $500 \mathrm{~nm}$-thick G-BHJ film (Supplementary Fig. 17 and Supplementary Table 14). Overall, the gradient polymer content is found to be more pronounced in thick G-BHJ OSCs (top: $16 \mathrm{wt} \%$; bottom: $90 \mathrm{wt} \%)$. Therefore, G-BHJ strategy is promising and feasible to construct highly efficient thick OSCs, critical to the future industrial roll-to-roll production. 

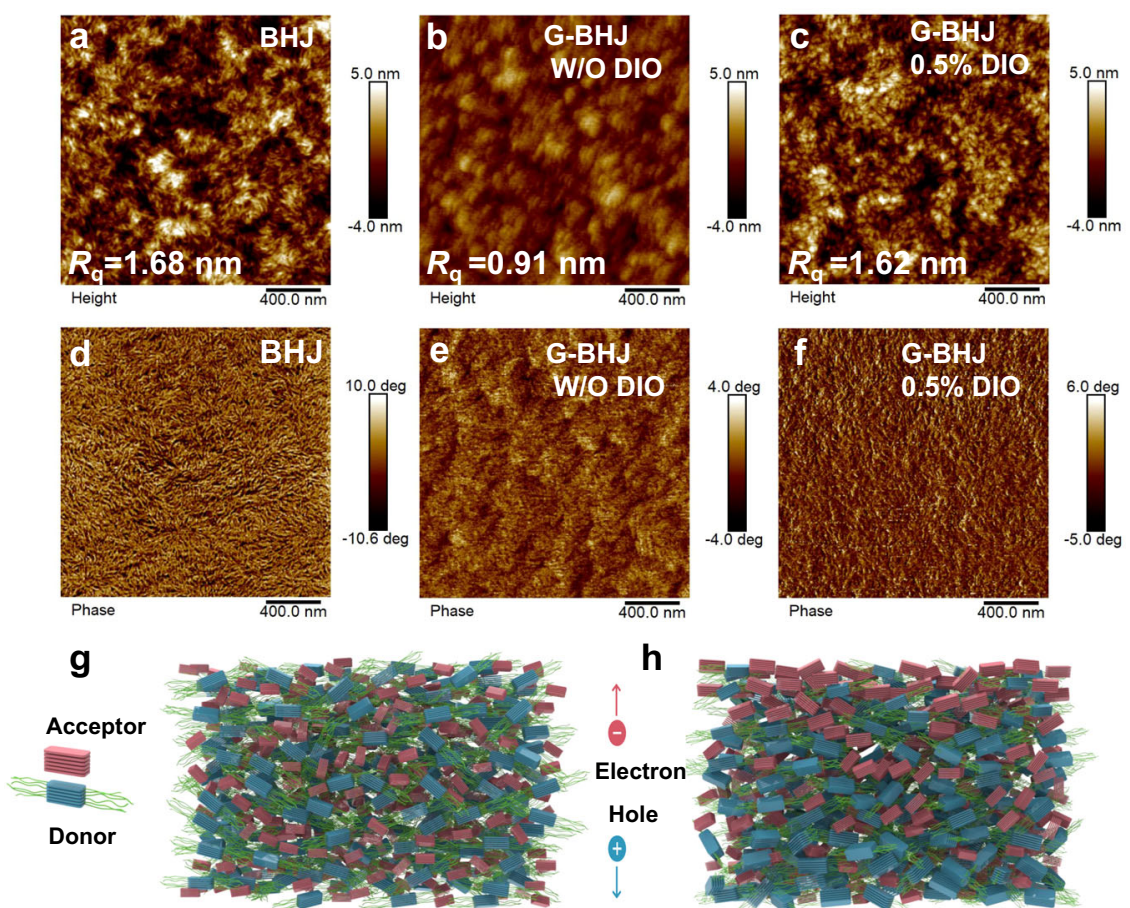

h

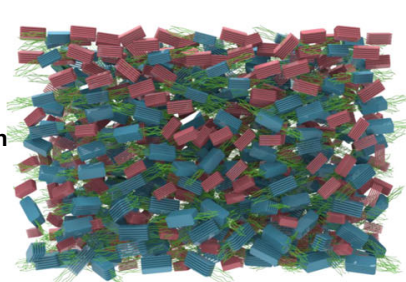

Fig. 4 The morphology of BHJ and G-BHJ films. a-c AFM height images, d-f AFM phase images of the optimal BHJ film, G-BHJ film without DIO and the optimal G-BHJ film with $0.5 \%$ DIO. The schematic morphology of $\mathbf{g}$ the optimal BHJ film and $\mathbf{h}$ the optimal G-BHJ film along the vertical direction.

a

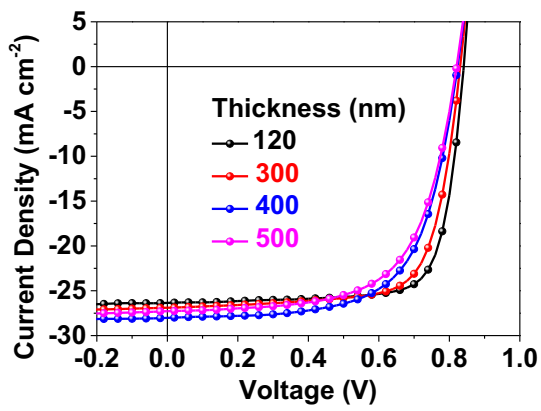

C

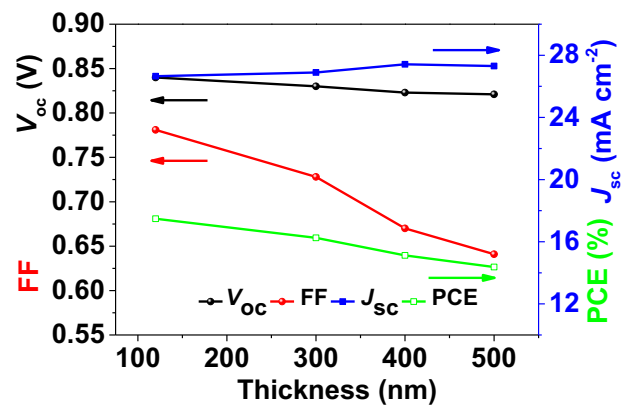

b

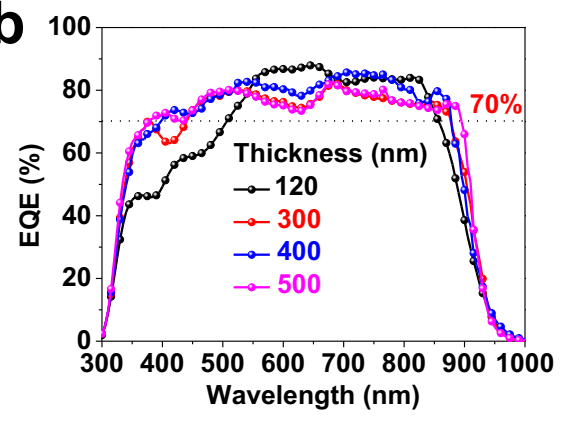

d

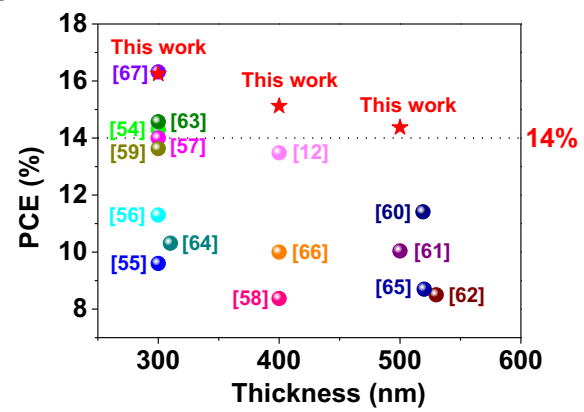

Fig. 5 The photovoltaic properties of thick G-BHJ OSCs. a J-V, b EQE curves of the optimal G-BHJ OSCs with different active layer thickness. c device performance parameters ( $P C E, J_{S C}, V_{O C}$, and FF) versus the active layer thickness. $\mathbf{d}$ Comparison of our results with previously reported PCEs and thickness for both binary and ternary OSCs. The detailed material systems and device parameters of each point are provided in Supplementary Table 12.

G-BHJ OSCs fabrication by blade coating. Encouraged by the outstanding photovoltaic performances of G-BHJ OSCs via spin coating method, we further employed doctor-blading technique to fabricate both $\mathrm{BHJ}$ and $\mathrm{G}-\mathrm{BHJ}$ OSCs in the same device structure, which is significant for the deployment of large-scale modules (Fig. 6a). In this study, BHJ and G-BHJ active layers was both deposited in open-air (30 $\pm 5 \% \mathrm{RH})$ using air-knife assisted blade-coating technique ${ }^{68,69}$, from non-halogenated $\mathrm{XY}$ solvent. The high boiling point of $\mathrm{XY}\left(140^{\circ} \mathrm{C}\right)$ will reinforce the aggregation $^{27,31}$, so employing laminar air-knife quenching together with hot-casting substrate at a moderate temperature of $60^{\circ} \mathrm{C}$ could well control the drying kinetics of polymer wet film. The active layers of both $\mathrm{BHJ}$ and G-BHJ are optimized by controlling DIO contents as well as the coating speeds (see 
Table 2 Photovoltaic parameters of the optimal G-BHJ OSCs with different active layer thickness.

\begin{tabular}{|c|c|c|c|c|c|}
\hline Active layer thickness (nm) & $v_{\text {oc }}(V)$ & $J_{\mathrm{SC}}\left(\mathrm{mA} \mathrm{cm} \mathrm{cm}^{-2}\right)$ & $\mathbf{F F}$ & $\mathrm{PCE}_{\max }\left(\mathrm{PCE}_{\mathrm{avg}}\right)^{\mathrm{a}}(\%)$ & $J_{\text {calc. }}{ }^{b}\left(\mathrm{~mA} \mathrm{~cm}{ }^{-2}\right)$ \\
\hline $120 \pm 5$ & 0.840 & 26.65 & 0.781 & $17.48(17.19 \pm 0.20)$ & 26.04 \\
\hline $300 \pm 10$ & 0.830 & 26.89 & 0.728 & $16.25(16.00 \pm 0.23)$ & 26.09 \\
\hline $400 \pm 10$ & 0.823 & 27.42 & 0.670 & $15.12(14.92 \pm 0.28)$ & 27.11 \\
\hline
\end{tabular}

The errors are defined as standard deviation.

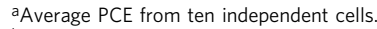

bdenotes integrated $J_{S C}$ from the EQE curves.

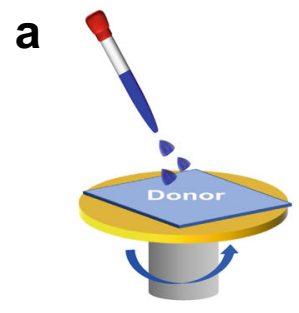

b

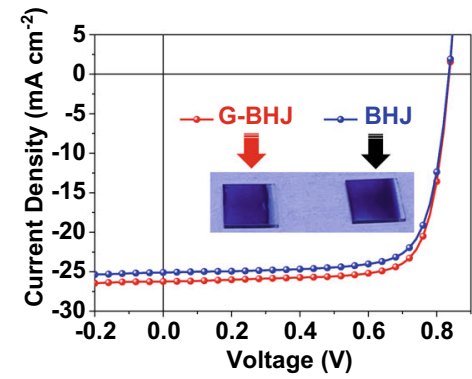

e
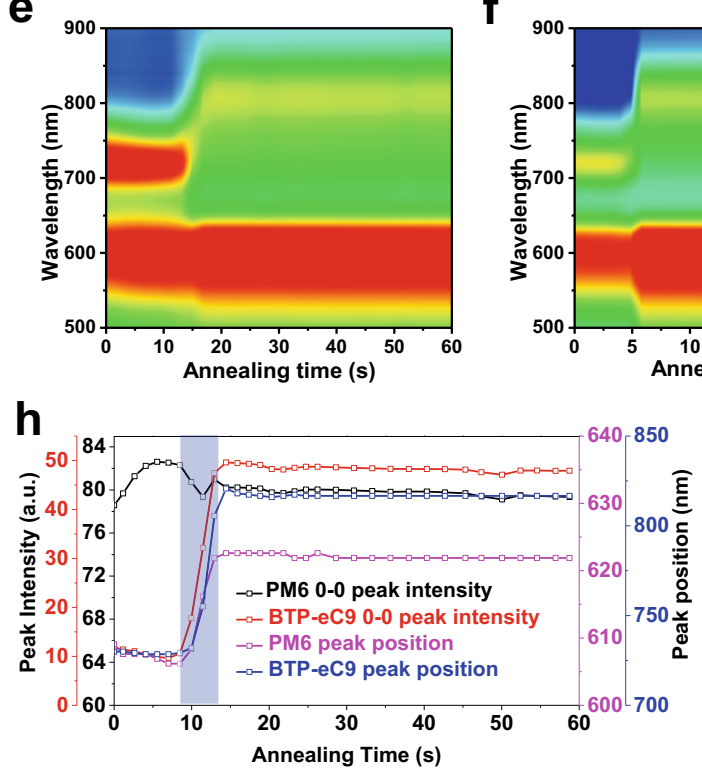

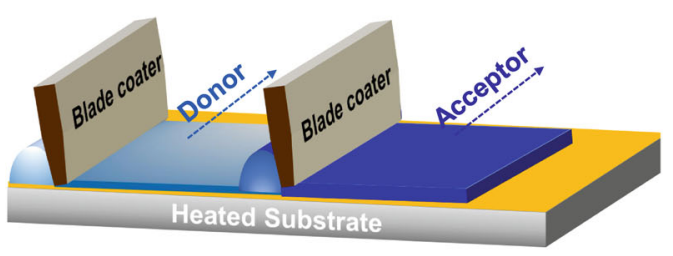

C

Blade coating

d
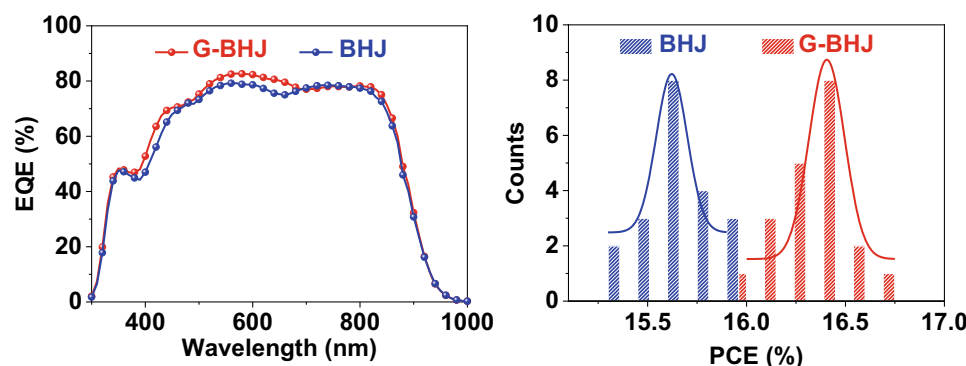

g
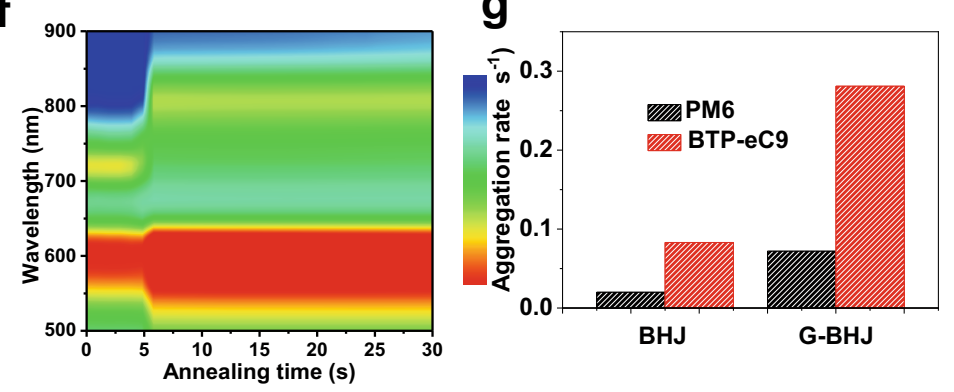

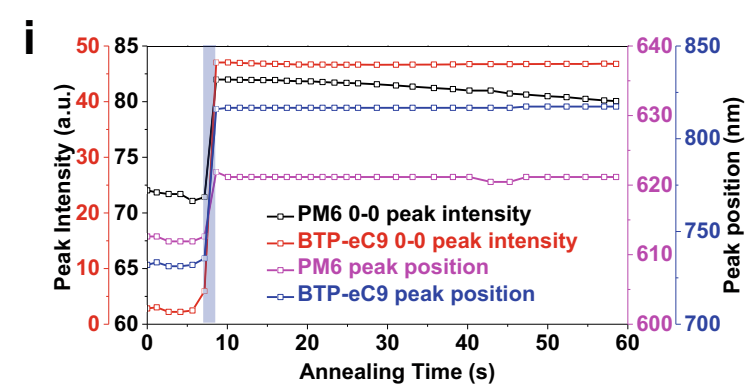

Fig. 6 The open-air blade coating device performances and in situ absorption characteristics for both BHJ and G-BHJ OSCs. a Schematic illustration of spin coating and blade coating for G-BHJ fabrication procedure. $\mathbf{b} J-V$ curves of the BHJ OSCs and G-BHJ OSCs. c EQE curves of the optimized BHJ OSCs and G-BHJ OSCs. d Histograms of the BHJ OSCs and G-BHJ OSCs based on 20 independent cells. Time-resolved UV-vis absorption spectra of e optimized $\mathrm{BHJ}$ and $\mathbf{f} \mathrm{G}-\mathrm{BHJ}$ OSCs films. $\mathbf{g}$ Aggregation rate of PM6 and BTP-eC9 in optimized BHJ and G-BHJ active layers. The peak position and peak intensity evolution as a function of annealing time of PM6 and BTP-eC9 in $\mathbf{h}$ optimized BHJ and $\mathbf{i}$ G-BHJ films. 
Supplementary Fig. 18 and Supplementary Tables 15, 16). Figure $6 \mathrm{~b}$ shows the $J-V$ curves of the optimized blade-coated $\mathrm{BHJ}$ and G-BHJ OSCs and the corresponding photovoltaic parameters are listed in the Table 1. The optimal G-BHJ devices exhibited a maximum PCE of $16.77 \%$ with a $V_{\mathrm{OC}}$ of $0.836 \mathrm{~V}$, a $J_{\mathrm{SC}}$ of $26.26 \mathrm{~mA} \mathrm{~cm}^{-2}$, and an FF of 0.764 . For blade-coated BHJ devices, an inferior efficiency of $15.87 \%$ was achieved accompanied by a $V_{\text {OC }}$ of $0.835 \mathrm{~V}$, a $J_{\text {SC }}$ of $25.24 \mathrm{~mA} \mathrm{~cm}^{-2}$ and an FF of 0.753 . The improvement of the photovoltaic performances in G-BHJ based device mainly originates from the enhanced $J_{\mathrm{SC}}$ and $\mathrm{FF}$, with the same trend as spin-coated devices. The higher $J_{\mathrm{SC}}$ value in G-BHJ device can be crosschecked with EQE measurements. The EQE spectra of G-BHJ device presented slightly higher photocurrent generation for both donor and acceptor absorption region. As a result, the integrated $J_{\mathrm{SCS}}$ are calculated from the EQE curves plotted in Fig. $6 \mathrm{c}$, to be 24.92 and $25.30 \mathrm{~mA} \mathrm{~cm}^{-2}$ for BHJ and G$\mathrm{BHJ}$ OSCs, respectively, which matches well with the $J-V$ measurements. The histograms of PCEs for BHJ and G-BHJ devices are plotted in Fig. 6d. The PCEs of G-BHJ OSCs are distributed within the range of 16.0 to $16.75 \%$, while the related $\mathrm{BHJ}$ devices fluctuated in the range of 15.3 to $15.9 \%$. We also investigated the CF-based G-BHJ OSCs via blade coating. As shown in Supplementary Fig. 7 and Supplementary Table 6, the optimal devices can give a high PCE of $16.78 \%$, which indicates the good compatibility of G-BHJ strategy in coating process. It is noteworthy that two other representative polymer/SMA material systems: PM6:IT-4F and PCE-10:IEICO-4F, gave better PCEs of G-BHJ versus their respective $\mathrm{BHJ}$ counterparts in both spin coating and blade coating processes, demonstrating the applicability of using G-BHJ strategy in D-A material combinations to promote photovoltaic performance of OSCs (see detailed performances in Supplementary Fig. 19 and Supplementary Table 17).

To understand the surprising enhancement of $J_{\mathrm{SC}}$ and $\mathrm{FF}$ in the G-BHJ devices processed by XY, we then investigated the crystallization kinetics of donor and acceptor. Here, in situ UV-vis absorption measurements were performed to detect the phase transition during the film formation from solution state to film state ${ }^{70}$. The $2 \mathrm{D}$ time-resolved UV-vis absorption mapping is displayed in Fig. 6e, $\mathrm{f}$ for $\mathrm{BHJ}$ and $\mathrm{G}-\mathrm{BHJ}$ films and the corresponding line profiles are provided in the Supporting Information. Since the absorption peak of donor and acceptor in the blends are clearly distinguishable, we then plotted the peak position and peak intensity of PM6 and BTP-eC9 as a function of annealing time to investigate their individual aggregation evolution (Fig. 6h, i). The $0-0$ absorption peaks of small molecule exhibited red-shift tendency from $\sim 731 \mathrm{~nm}$ to $\sim 820 \mathrm{~nm}$ in both $\mathrm{BHJ}$ and $\mathrm{G}-\mathrm{BHJ}$ films accompanied by a dramatic increase of peak intensity, which implies that the molecular ordering stage of BTP-eC9 occurs (Supplementary Fig. 20). Noticeably, it takes $12.9 \mathrm{~s}$ for the $\mathrm{BHJ}$ film to form a dry film, while it takes only $8.6 \mathrm{~s}$ for the G-BHJ film to be solidified under the same post-treatment condition, implying that the G-BHJ strategy effectively facilitates the film drying process. Interestingly, another distinction between $\mathrm{G}-\mathrm{BHJ}$ and $\mathrm{BHJ}$ films exists in the wet film stage. While the peak intensity of polymer in BHJ film (black curve in Fig. 6h) undergoes an increased trend due to the evaporation of solvent (so called pre-aggregation stage), it is completely suppressed in the G-BHJ film, indicating that the G-BHJ processing strategy affects the aggregation of polymers and thus lead to the distinctive morphological features. We infer that when depositing the second acceptor layer, the bottom polymer layer is actually entering the semi-wet state, thus the pre-aggregation stage is ignored and directly undergoes the film transformation from semi-wet state to the solid state. Furthermore, the aggregation rate of polymer and small molecule was deduced from the slope of the peak and studied, shown in Fig. 6g. It was found that the aggregation rate of small molecule is higher than that of polymer in both $\mathrm{BHJ}$ $\left(0.083 \mathrm{~s}^{-1}\right.$ for acceptor and $0.020 \mathrm{~s}^{-1}$ for donor) and G-BHJ films $\left(0.281 \mathrm{~s}^{-1}\right.$ for acceptor and $0.072 \mathrm{~s}^{-1}$ for donor). Compared to the aggregation rate of $\mathrm{BTP}-\mathrm{eC} 9$ in $\mathrm{BHJ}$ film, the acceptor in the G-BHJ film can be rapidly aggregated which is indicated by the sharp slope of the peak, almost three times faster than that in $\mathrm{BHJ}$ film. We hypothesize that the polymer layer could offer nuclei (or nucleation sites) for the small molecule layer in the G-BHJ process, which could induce the faster ordering of the small molecule layer ${ }^{29}$. It has revealed that the slow solidification leads to longer time for the active layer to aggregate and crystallize, giving oversized domain or excessive phase separation in the active layer ${ }^{46}$. Therefore, the faster film formation process in the G-BHJ provides rapid crystallization time, which prohibits the excessive aggregation of $\mathrm{NFA}^{30}$. Moreover, the aggregation rate of polymer in the G-BHJ film also becomes higher in comparison with that in the $\mathrm{BHJ}$ film. This faster polymer aggregation rate is associated with the inhibited polymer pre-aggregation stage, which saves time for the semi-solid film to transform directly to the solid film. The more balanced aggregation kinetics between the polymer and small molecule NFA in the G-BHJ blend indicate the more balanced phase domains, which is beneficial to efficient exciton dissociation and charge transport, contributing to higher $J_{\mathrm{SC}}$ and FF. We also performed AFM measurements to probe the surface morphology of the optimal blade-coated BHJ and G-BHJ films (Supplementary Fig. 21). As expected, small RMS value in the optimal G-BHJ film $(1.14 \mathrm{~nm})$ can be observed, confirming the suppressed aggregation behavior in the G-BHJ printed active layer. Therefore, we have validated the non-halogenated solvent enabled G-BHJ method for highly efficient blade-coated OSCs via regulating the crystallization kinetics of both donor and acceptor and thus phase separation in the active layer.

\section{Discussion}

In this manuscript, we demonstrate the G-BHJ morphology modulation in state-of-the-art OSC systems with proposed solvent guidance. Insights of G-BHJs are provided through quantitative investigations of depth-profiling XPS and angle-dependent GI-XRD techniques. The graded composition and crystallinity distribution in principle can benefit the carrier transport, while the graded $\mathrm{BHJ}$ still gives sufficient $\mathrm{D} / \mathrm{A}$ interface. The spincoated G-BHJ OSCs processed by $\mathrm{CF}$ and XY deliver outstanding PCEs of $17.54 \%$ and $17.48 \%$, respectively. The G-BHJs' carrier transport advantage indeed enabled high-performance thick film OSCs from XY-16.25\% (FF of 0.728 ) for $300 \mathrm{~nm}, 15.12 \%$ (FF of 0.670 ) for $400 \mathrm{~nm}$, and $14.37 \%$ (FF of 0.641 ) for $500 \mathrm{~nm}$ thick OSCs. Probably even more excitingly, the nonhalogenated solvent (XY) enabled G-BHJ OSC via blade coating to achieve an excellent $16.77 \%$ PCE in open air condition, assisted by the drastically different but favorable novel D-A crystallization kinetics comparing to classical $\mathrm{BHJ}$.

One should remember that these encouraging results were achieved using proper solvent coating twice sequentially (CF and $\mathrm{XY}$ as examples here). This is just at the infant stage of a new research direction, and it opens a door of solvent engineering for achieving various $\mathrm{G}-\mathrm{BHJ}$ with tunable D/A composition and crystallinity gradient profiles. In our opinion, smart selection of "green" solvents with different solubilities to the existing high performance OSC systems and engineering deposition conditions wisely, or even design new OSC D-A molecules fitting "good" solvents are both very valuable for next step G-BHJ OSC research. The scale-up effort is always an important step for the scientific findings to be applicable. The in situ approaches, including the $\mathrm{UV}-\mathrm{V}$ is here as well as GI-XRD, electron microscopy etc., are believed to be critical for understanding the solar ink thin film 
molecular packing, morphology evolution and structure-property relationship. These are challenging tasks, but deserve serious investigations to move the new field forward. Overall, this work shows the G-BHJ reported here is a feasible and promising strategy towards highly efficient, ecofriendly and manufacture friendly OSCs.

\section{Methods}

Materials. The molecular weight of the PM6 was characterized with high temperature gel permeation chromatography (GPC) at $160^{\circ} \mathrm{C}$ with $1,2,4$-trichlorobenzene as solvent, which yields $M_{\mathrm{n}}=35,800, M_{\mathrm{w}}=97,000$, PDI $=2.7$. BTP-eC9 were purchased from Solarmer Materials Inc. All other chemical reagents were used as received.

Device fabrication and characterization. Polymer solar cell devices were fabricated in the conventional structure of ITO/PEDOT: PSS/Active layer/PFN-Br/Ag. The substrates were firstly cleaned using detergent, deionized water, acetone, and isopropanol for every $30 \mathrm{~min}$, and then treated in ultraviolet ozone for $20 \mathrm{~min}$. Followed by spin coating a thin layer $(\sim 30 \mathrm{~nm})$ of PEDOT:PSS (Bayer baytron 4083 ) on the precleaned ITO-coated glass substrates at $3000 \mathrm{rpm}$ for $30 \mathrm{~s}$ and then annealed at $150{ }^{\circ} \mathrm{C}$ for $15 \mathrm{~min}$. Then, the substrates were transferred into a glovebox. Afterwards, for the BHJ devices, the optimized active layer was spin coated from $o$-xylene solution with polymer concentration of $10 \mathrm{mg} \mathrm{mL}^{-1}$ (D:A $=1: 1.2$, $0.5 \%$ DIO) at $3500 \mathrm{rpm}$ for $60 \mathrm{~s}$ to form an active layer of $\sim 120 \mathrm{~nm}$. Then, the active layer was thermal annealed at $100^{\circ} \mathrm{C}$ for $10 \mathrm{~min}$ on the hotplate. For the GBHJ processed device, the PM6 solution was prepared in $o$-xylene at $10 \mathrm{mg} \mathrm{mL}^{-1}$ at $60^{\circ} \mathrm{C}$ stirring. BTP-eC9 solution was prepared in $o$-xylene at $10 \mathrm{mg} \mathrm{mL}^{-1}$ with $0.5 \%$ DIO by volume at $80^{\circ} \mathrm{C}$ stirring. The PM6 solution was spin-coated on PEDOT:PSS substrates at $1750 \mathrm{rpm}$ for $60 \mathrm{~s}$ to obtain the donor layer of about $70 \mathrm{~nm}$. The BTP-eC9 solution was spin-coated on the rotating PM6 donor layer at $2250 \mathrm{rpm}$ for $60 \mathrm{~s}$ to obtain the acceptor layers of about $55 \mathrm{~nm}$. Then, the active layers were treated with thermal annealing at $100^{\circ} \mathrm{C}$ for $10 \mathrm{~min}$. As for BHJ cells fabricated via spin coating using $\mathrm{CF}$, the active layer was spin coated from $16.5 \mathrm{mg}$ $\mathrm{mL}^{-1} \mathrm{CF}$ solution (D:A $=1: 1.2,0.5 \% \mathrm{v} / \mathrm{v}$ 1-chloronaphthalene, $\mathrm{CN}$ ) at $3750 \mathrm{rpm}$ for $30 \mathrm{~s}$. For G-BHJ devices, the donor was firstly deposited from $7.25 \mathrm{mg} \mathrm{mL}^{-1} \mathrm{CF}$ solution at $3000 \mathrm{rpm}$ for $30 \mathrm{~s}$, followed by deposing the acceptor solution in $7.5 \mathrm{mg}$ $\mathrm{mL}^{-1}$ with $0.5 \% \mathrm{v} / \mathrm{v} \mathrm{CN}$ in acceptor solution at $2750 \mathrm{rpm}$ for $30 \mathrm{~s}$. Then, a thermal annealing treatment at $100^{\circ} \mathrm{C}$ for $10 \mathrm{~min}$ was performed for both active layers. For thicker film fabrication for the BHJ and G-BHJ OSCs, the variation of thickness was controlled by changing the total concentration and the spin coating speed. To be detailed, for $\mathrm{BHJ}$ thick devices, the $300 \mathrm{~nm}$ thickness was given by spin coating the solution of $15 \mathrm{mg} \mathrm{mL}^{-1}$ at the spin speed of $2750 \mathrm{rpm}, 400 \mathrm{~nm}$, and $500 \mathrm{~nm}$ thicknesses were given by spin coating the solution of $20 \mathrm{mg} \mathrm{mL}^{-1}$ at the spin speed of $4000 \mathrm{rpm}$ and $3000 \mathrm{rpm}$, respectively. For G-BHJ thick devices, the concentrations of donor and acceptor (D/A) are $18 / 18 \mathrm{mg} \mathrm{mL}^{-1}, 300 \mathrm{~nm}$-based active layer was realized at the speed of $1750 / 1750 \mathrm{rpm}$ for $\mathrm{D} / \mathrm{A}, 20 / 20 \mathrm{mg} \mathrm{mL}^{-1}$ of D/A can get $400 \mathrm{~nm}$-thick and $500 \mathrm{~nm}$-thick active layers at $3250 \mathrm{rpm} / 1750 \mathrm{rpm}$ and $2750 / 2750 \mathrm{rpm}$, respectively. Then a thermal annealing at $100^{\circ} \mathrm{C}$ for $10 \mathrm{~min}$ was performed. The thicknesses of the films were measured by a Bruker Dektak XT stylus profilometer. For the blade-coated devices, PM6 and BTP-eC9 were dissolved at the polymer concentration of $10 \mathrm{mg} \mathrm{mL}^{-1}$ and the acceptor concentration of $10 \mathrm{mg} \mathrm{mL}^{-1}$. Before blade-coating the active layer, $0.1 \%, 0.3 \%$, and $0.5 \% 1,8$ diiodooctane $(\mathrm{v} / \mathrm{v})$ was added into acceptor layer solutions. An automatic wire-bar coater (RK PrintCoat Instruments, K paint type) was empolyed in a humiditycontrol ambient environment $(30 \pm 5 \% \mathrm{RH})$. Small droplet of active layer solution was dripped on the substrate and swiped linearly by an adjustable film applicator (BEVS 1806B/100) The blade speed was set as 20, 25, 30, 40,50, and $60 \mathrm{~mm} \mathrm{~s}^{-1}$. The gap between the film applicator and substrate was set as $100 \mu \mathrm{m}$. When coating the active layer, the ITO-based substrate was maintained as $60^{\circ} \mathrm{C}$. A laminar nitrogen knife was installed right next to the substrate with the flow at an angle of $20^{\circ}$ to the substrate. The as-prepared wet active layer film was gas-quenched by the nitrogen knife with the fixed nitrogen blow rate of $40 \mathrm{~m} \mathrm{~s}^{-1}$ (calibrated using the Testo 416 flowmeter), in order to facilitate solvent drying and film crystallization. Subsequently, the films were treated with the thermal annealing at $100^{\circ} \mathrm{C}$ for 10 min.

For the optimal BHJ devices via blade coating, $16.5 \mathrm{mg} \mathrm{mL}^{-1} \mathrm{CF}$ solution $(D$ : $A=1: 1.2,0.5 \% \mathrm{v} / \mathrm{v} \mathrm{CN}$ ) was blade coated at the blade speed of $25 \mathrm{~mm} \mathrm{~s}^{-1}$. For the optimal G-BHJ devices via blade coating, $7.25 \mathrm{mg} \mathrm{mL}^{-1} \mathrm{CF}$ solution of donor was blade coated at the blade speed of $25 \mathrm{~mm} \mathrm{~s}^{-1}, 7.25 \mathrm{mg} \mathrm{mL}^{-1} \mathrm{CF}$ solution of acceptor $(0.5 \% \mathrm{v} / \mathrm{v} \mathrm{CN})$ was blade coated at the blade speed of $60 \mathrm{~mm} \mathrm{~s}^{-1}$. When coating the active layer, the ITO-based substrate was maintained at room temperature. The as-prepared wet active layer film was gas-quenched by the nitrogen knife with the fixed nitrogen blow rate of $40 \mathrm{~m} \mathrm{~s}^{-1}$. Then, a thermal annealing of $100^{\circ} \mathrm{C}$ for $10 \mathrm{~min}$ was performed for all the films.

Deposition of interface layer in the blade-coated devices are the same as that in the spin-coated devices. As for G-BHJ OSCs based on PM6:IT-4F and PCE-10: IEICO- $4 \mathrm{~F}$ systems, optimal active layers were spin-coated from XY solutions with a
D:A weight ratio of 1:1 for PM6:IT-4F and 1:1.5 for PCE-10:IEICO-4F with a small amount of additive in acceptor solutions $(0.5 \%$ DIO for IT- $4 \mathrm{~F}, 2 \% \mathrm{CN}$ for IEICO$4 \mathrm{~F}$ ), followed by a post thermal annealing of $100^{\circ} \mathrm{C}$ for $10 \mathrm{~min}$. A thin layer $(\sim 5 \mathrm{~nm})$ of PFN-Br was spin coated on the active layer at $3000 \mathrm{rpm}$ for $30 \mathrm{~s}$. Finally, the $\mathrm{Ag}(90 \mathrm{~nm})$ electrode was deposited by thermal evaporation to complete the whole device with the active area of $0.04 \mathrm{~cm}^{2}$, which is typical defined by a metal mask with an aperture aligned with the device area.

The current density-voltage $(J-V)$ characteristics were measured in the glovebox with a Keithley 2400 measure unit under 1 sun, AM $1.5 \mathrm{G}$ spectra $\left(100 \mathrm{~mW} \mathrm{~cm}^{-2}\right)$ from a solar simulator (Enli Tech. Co., Ltd., Taiwan). The light intensity was calibrated with a $20 \mathrm{~mm} \times 20 \mathrm{~mm}$ monocrystalline silicon reference cell with KG5 filter (Enli Tech. Co., Ltd., Taiwan). The $J-V$ curves are measured along the forward scan direction from -1.5 to $1.5 \mathrm{~V}$, with a scan step of $20 \mathrm{mV}$ with delay time of $1 \mathrm{~ms}$. The EQE was measured by solar cell spectral response measurement system QE-R3-011 (Enli Tech. Co., Ltd., Taiwan). The light intensity at each wavelength was calibrated with a standard single-crystal Si photovoltaic cell.

DP-XPS measurements. XPS experiments performed on the Thermo Scientific Nexsa using $12 \mathrm{kV}$ cathode biased $\mathrm{Al} \mathrm{Ka}$ radiative source. The base pressure in the analysis chamber was about $5 \times 10^{-10}$ mbar. Depth profiling tests were conducted by using an $\mathrm{Ar}^{+}$sputtering gun operated at $1 \mathrm{keV}$ with raster size of $1 \mathrm{~mm} \times 1 \mathrm{~mm}$.

TOF-SIMS measurements. TOF-SIMS was performed using a TOF-SIMS instrument (ION TOF TOF-SIMS V), where a $10 \mathrm{keV} \mathrm{Ar}_{1500}+$ cluster ion source was used for sputtering and a $25 \mathrm{keV} \mathrm{Bi}_{3}{ }^{+}$pulsed primary ion beam was used for the analysis. The area of analysis was $150 \times 150 \mu \mathrm{m}^{2}$.

SCLC measurements. The hole and electron mobility of the D/A blend film was measured using the space charge limited current (SCLC) method. The structure of hole-only device and electron-only mobility device is ITO/PEDOT: PSS/Active layer/Au and ITO/ZnO/ Active layer/Ag, respectively. The mobility was determined by fitting the dark current to the model of a single carrier SCLC, which is described by the equation:

$$
J=\frac{9}{8} \varepsilon_{0} \varepsilon_{\mathrm{r}} \mu_{\mathrm{h}} \frac{V^{2}}{d^{3}}
$$

where $J$ is the current, $\mu_{\mathrm{h}}$ is the charge mobility at zero field, $\varepsilon_{\mathrm{o}}$ is the free-space permittivity, $\varepsilon_{\mathrm{r}}$ is the relative permittivity of the material, $d$ is the thickness of the active layer, and $V$ is the effective voltage $V-V_{b \text { bi }}$, where is the built-in voltage. The hole-mobility and electron-mobility can be fit from the $\operatorname{Ln}\left(\Omega^{3} / V^{2}\right)-(V / L)^{0.5}$ curves and $\operatorname{Ln}(J)-\operatorname{Ln}(V)$ curves respectively.

GI-XRD characterization. GI-XRD measurements were carried out with a Xeuss 2.0 SAXS/WAXS laboratory beamline using a Cu X-ray source $(8.05 \mathrm{keV}, 1.54 \AA)$ and Pilatus $3 \mathrm{R} 300 \mathrm{~K}$ detector. The critical incidence angle is $0.2^{\circ}$. For the angledependent GI-XRD, $0.15^{\circ}, 0.20^{\circ}$, and $0.25^{\circ}$ were chosen.

Photoluminescence characterizations. The steady PL spectra were measured by the FLS 920 (Edinburgh Instruments, Ltd) with excitation at $635 \mathrm{~nm}$.

Reporting summary. Further information on research design is available in the Nature Research Reporting Summary linked to this article.

\section{Data availability}

The authors declare that the experimental data that support the findings of this paper are available within the article and its Supplementary Information files. The source data are provided as a Source Data file. Other findings in this study are available from the corresponding authors upon reasonable request. Source data are provided with this paper.

Received: 8 February 2021; Accepted: 26 July 2021; Published online: 10 August 2021

\section{References}

1. Li, G., Zhu, R. \& Yang, Y. Polymer solar cells. Nat. Photonics 6, 153-161 (2012)

2. Li, G., Chu, C. W., Shrotriya, V., Huang, J. \& Yang, Y. Efficient inverted polymer solar cells. Appl. Phys. Lett. 88, 253503 (2006).

3. Liang, Y. et al. For the bright future-bulk heterojunction polymer solar cells with power conversion efficiency of 7.4\%. Adv. Mater. 22, 135-1388 (2010).

4. Chang, S.-Y., Cheng, P., Li, G. \& Yang, Y. Transparent polymer photovoltaics for solar energy harvesting and beyond. Joule 2, 1039-1054 (2018). 
5. Li, G. et al. High-efficiency solution processable polymer photovoltaic cells by self-organization of polymer blends. Nat. Mater. 4, 864-868 (2005).

6. Liu, D., Zhang, Y. \& Li, G. Nanomorphology in A-D-A type small molecular acceptors-based bulk heterojunction polymer solar cells. J. Energy Chem. 35, 104-123 (2019).

7. Lin, Y. et al. An electron acceptor challenging fullerenes for efficient polymer solar cells. Adv. Mater. 27, 1170-1174 (2015).

8. Zhao, W. et al. Fullerene-free polymer solar cells with over $11 \%$ efficiency and excellent thermal stability. Adv. Mater. 28, 4734-4739 (2016).

9. Hou, J., Inganas, O., Friend, R. H. \& Gao, F. Organic solar cells based on nonfullerene acceptors. Nat. Mater. 17, 119-128 (2018).

10. Huang, J., Tang, H., Yan, C. \& Li, G. 1,1-Dicyanomethylene-3-indanone endcap engineering for fused-ring electron acceptor-based high-performance organic photovoltaics. Cell Rep. Phys. Sci. 2, 10092 (2021).

11. Zhang, Y., Liu, D., Fong, P. W. K. \& Li, G. Investigation of low-bandgap nonfullerene acceptor-based polymer solar cells with very low photovoltage loss. J. Photon. Energy 9, 045502 (2019).

12. Zhan, L. et al. Over $17 \%$ efficiency ternary organic solar cells enabled by two non-fullerene acceptors working in alloy-like model. Energy Environ. Sci. 13, 635-645 (2020).

13. Cui, Y. et al. Single-iunction organic photovoltaic cells with approaching $18 \%$ efficiency. Adv. Mater. 32, 1908205 (2020).

14. Yao, J. et al. Cathode engineering with perylene-diimide interlayer enabling over 17\% efficiency single-junction organic solar cells. Nat. Commun. 11, 2726 (2020).

15. Lin, Y. et al. $17 \%$ Efficient organic solar cells based on liquid exfoliated WS2 as a replacement for PEDOT:PSS. Adv. Mater. 31, 1902965 (2019).

16. Lin, Y. et al. Self-assembled monolayer enables hole transport layer-free organic solar cells with $18 \%$ efficiency and improved operational stability. ACS Energy Lett. 5, 2935-2944 (2020).

17. Li, G. et al. "Solvent annealing" effect in polymer solar cells based on poly(3hexylthiophene) and methanofullerenes. Adv. Funct. Mater. 17, 1636-1644 (2007).

18. Li, S. et al. Energy-level modulation of small-molecule electron acceptors to achieve over $12 \%$ efficiency in polymer solar cells. Adv. Mater. 28, 9423-9429 (2016).

19. Yan, Y., Liu, X. \& Wang, T. Conjugated-polymer blends for organic photovoltaics: rational control of vertical stratification for high performance. Adv. Mater. 29, 1601674 (2017).

20. Ye, L. et al. High-efficiency nonfullerene organic solar cells: critical factors that affect complex multi-length scale morphology and device performance. Adv. Energy Mater. 7, 1602000 (2017).

21. Ye, L. et al. Quantitative relations between interaction parameter, miscibility and function in organic solar cells. Nat. Mater. 17, 253-260 (2018).

22. Jiang, K. et al. Alkyl chain tuning of small molecule acceptors for efficient organic solar cells. Joule 3, 3020-3033 (2019).

23. Liu, T. et al. A nonfullerene acceptor with a $1000 \mathrm{~nm}$ absorption edge enables ternary organic solar cells with improved optical and morphological properties and efficiencies over 15\%. Energy Environ. Sci. 12, 2529-2536 (2019).

24. Liao, X. et al. Non-halogenated solvent-processed single-junction polymer solar cells with $9.91 \%$ efficiency and improved photostability. Nano Energy 41, 27-34 (2017)

25. Ma, L. et al. High efficiency non-fullerene organic solar cell enabled by 1000$\mathrm{nm}$-thick active layers with a low trap-state density. ACS Appl. Mater. Interfaces 12, 18777-18784 (2020).

26. Pan, F. et al. As-cast ternary polymer solar cells based on a nonfullerene acceptor and its fluorinated counterpart showing improved efficiency and good thickness tolerance. J. Mater. Chem. A 7, 9798-9806 (2019).

27. Zhao, W. et al. Environmentally friendly solvent-processed organic solar cells that are highly efficient and adaptable for the blade-coating method. Adv. Mater. 30, 1704837 (2018)

28. Sun, R. et al. A layer-by-layer architecture for printable organic solar cells overcoming the scaling lag of module efficiency. Joule 4, 407-419 (2020).

29. Wang, Y. et al. Achieving balanced crystallization kinetics of donor and acceptor by sequential-blade coated double bulk heterojunction organic solar cells. Adv. Energy Mater. 10, 2000826 (2020).

30. Lin, B. et al. Balancing the pre-aggregation and crystallization kinetics enables high efficiency slot-die coated organic solar cells with reduced non-radiative recombination losses. Energy Environ. Sci. 13, 2467-2479 (2020).

31. Zhao, W. et al. Vacuum-assisted annealing method for high efficiency printable large-area polymer solar cell modules. J. Mater. Chem. C 7, 3206-3211 (2019).

32. Nelson, J. The Physics of Solar Cells (Imperial College Press, London, 2003).

33. Li, X. et al. Layer-by-layer solution processing method for organic solar cells. Sol. RRL 5, 2000592 (2020)

34. Liu, Y. et al. Sequential deposition: optimization of solvent swelling for highperformance polymer solar cells. ACS Appl. Mater. Interfaces 7, 653-661 (2015).
35. Ye, L. et al. Sequential deposition of organic films with eco-compatible solvents improves performance and enables over $12 \%$-efficiency nonfullerene solar cells. Adv. Mater. 31, 1808153 (2019).

36. Dong, S. et al. High-performance large-area organic solar cells enabled by sequential bilayer processing via nonhalogenated solvents. Adv. Energy Mater. 9, 1802832 (2019).

37. Cheng, P. et al. Efficient and stable organic solar cells via a sequential process J. Mater. Chem. C 4, 8086-8093 (2016).

38. Kumar, A., Li, G., Hong, Z. \& Yang, Y. High efficiency polymer solar cells with vertically modulated nanoscale morphology. Nanotechnology 20, 165202 (2009).

39. Lin, Y. et al. Small-molecule solar cells with fill factors up to 0.75 via a layerby-layer solution process. Adv. Energy Mater. 4, 1300626 (2014).

40. Wang, Y. \& Zhan, X. Layer-by-layer processed organic solar cells. Adv. Energy Mater. 6, 1600414 (2016).

41. Cui, Y. et al. Toward efficient polymer solar cells processed by a solutionprocessed layer-by-layer approach. Adv. Mater. 30, 1802499 (2018).

42. Sun, R. et al. A multi-objective optimization-based layer-by-layer bladecoating approach for organic solar cells: rational control of vertical stratification for high performance. Energy Environ. Sci. 12, 3118-3132 (2019).

43. Zhang, X. et al. Molecular dispersion enhances photovoltaic efficiency and thermal stability in quasi-bilayer organic solar cells. Sci. China Chem. 64, 116-126 (2021).

44. Fu, H. et al. A generally applicable approach using sequential deposition to enable highly efficient organic solar cells. Small Methods 4, 2000687 (2020).

45. Zhan, L. et al. Layer-by-layer processed ternary organic photovoltaics with efficiency over 18. Adv. Mater. 33, 2007231 (2021).

46. Dong, S. et al. Suppressing the excessive aggregation of nonfullerene acceptor in blade-coated active layer by using $n$-type polymer additive to achieve largearea printed organic solar cells with efficiency over 15\%. EcoMat 1, 12006 (2019).

47. Rasool, S. et al. Room temperature processed highly efficient large-area polymer solar cells achieved with molecular engineering of copolymers. Adv. Energy Mater. 9, 1900168 (2019).

48. Dong, S., Jia, T., Zhang, K., Jing, J. \& Huang, F. Single-component nonhalogen solvent-processed high-performance organic solar cell module with efficiency over 14\%. Joule 4, 2004-2016 (2020).

49. Li, M., Wang, Q., Liu, J., Geng, Y. \& Ye, L. Sequential deposition enables highperformance nonfullerene organic solar cells. Mater. Chem. Front. 5, 4851-4873 (2021).

50. Cheng, P. et al. Ternary system with controlled structure: a new strategy toward efficient organic photovoltaics. Adv. Mater. 30, 1705243 (2018).

51. Li, Q. et al. Vertical composition distribution and crystallinity regulations enable high-performance polymer solar cells with $>17 \%$ efficiency. ACS Energy Lett. 5, 3637-3646 (2020).

52. Qin, M. et al. Precise control of perovskite crystallization kinetics via sequential A-site doping. Adv. Mater. 32, 2004630 (2020).

53. Xiao, Y. \& Lu, X. Morphology of organic photovoltaic non-fullerene acceptors investigated by grazing incidence X-ray scattering techniques. Mater. Today Nano 5, 100030 (2019).

54. Ma, L. et al. A ternary organic solar cell with $300 \mathrm{~nm}$ thick active layer shows over 14\% efficiency. Sci. China Chem. 63, 21-27 (2019).

55. Tang, H. et al. Delicate morphology control triggers $14.7 \%$ efficiency all-smallmolecule organic solar cells. Adv. Energy Mater. 10, 2001076 (2020).

56. Wang, J. L. et al. Selenopheno[3,2-b]thiophene-based narrow-bandgap nonfullerene acceptor enabling $13.3 \%$ efficiency for organic solar cells with thickness-insensitive feature. ACS Energy Lett. 3, 2967-2976 (2018).

57. Zhang, Y. et al. A novel wide-bandgap polymer with deep ionization potential enables exceeding $16 \%$ efficiency in ternary nonfullerene polymer solar cells. Adv. Funct. Mater. 30, 1910466 (2020).

58. Zhang, G. et al. High-performance ternary organic solar cell enabled by a thick active layer containing a liquid crystalline small molecule donor. J. Am. Chem. Soc. 139, 2387-2395 (2017).

59. Zhou, Z. et al. High-efficiency small-molecule ternary solar cells with a hierarchical morphology enabled by synergizing fullerene and non-fullerene acceptors. Nat. Energy 3, 952-959 (2018).

60. Zhang, Y. et al. High performance thick-film nonfullerene organic solar cells with efficiency over $10 \%$ and active layer thickness of $600 \mathrm{~nm}$. Adv. Energy Mater. 9, 1902688 (2019)

61. Gao, W. et al. Thick-film organic solar cells achieving over $11 \%$ efficiency and nearly $70 \%$ fill factor at thickness over $400 \mathrm{~nm}$. Adv. Funct. Mater. 30, 1908336 (2020).

62. Li, S. et al. Design of a new small-molecule electron acceptor enables efficient polymer solar cells with high fill factor. Adv. Mater. 29, 1704051 (2017).

63. Gao, J. et al. Over $14.5 \%$ efficiency and $71.6 \%$ fill factor of ternary organic solar cells with $300 \mathrm{~nm}$ thick active layers. Energy Environ. Sci. 13, 958-967 (2020).

64. Sun, C. et al. A low cost and high performance polymer donor material for polymer solar cells. Nat. Commun. 9, 743 (2018). 
65. Li, Z.-Y. et al. Achieving efficient thick film all-polymer solar cells using a green solvent additive. Chin. J. Polym. Sci. 38, 323-331 (2019).

66. Luo, Z. et al. Side-chain impact on molecular orientation of organic semiconductor acceptors: high performance nonfullerene polymer solar cells with thick active layer over $400 \mathrm{~nm}$. Adv. Energy Mater. 8, 1800856 (2018).

67. Qin, J. et al. Over $16 \%$ efficiency from thick-film organic solar cells. Sci. Bull. 65, 1979-1982 (2020).

68. Liu, K. et al. Zwitterionic-surfactant-assisted room-temperature coating of efficient perovskite solar cells. Joule 4, 2404-2425 (2020).

69. $\mathrm{Hu}, \mathrm{H}$. et al. Room-temperature meniscus coating of $>20 \%$ perovskite solar cells: a film formation mechanism investigation. Adv. Funct. Mater. 29, 1900092 (2019).

70. Fong, P. W. K. et al. Printing high-efficiency perovskite solar cells in highhumidity ambient environment-an in situ guided investigation. Adv. Sci. 8, 2003359 (2021).

\section{Acknowledgements}

G.L. and K.L. thanks the Research Grants Council of Hong Kong (GRF grant 15218517, CRF C5037-18G, PDFS2021-5S04), National Science Foundation of China (NSFC

51961165102), the funding support from Shenzhen Science and Technology Innovation Commission (Project No. JCYJ 20200109105003940), the Sir Sze-yuen Chung Endowed Professorship Fund (8-8480) provided by the Hong Kong Polytechnic University, and K. L. thanks Guangdong Basic Research Foundation (2020A1515110156). X.L. thanks the Research Grants Council of Hong Kong (GRF grant 14314216).

\section{Author contributions}

G.L. convinced the idea. Y.Zhang and K.L. performed the device fabrication and char acterization, P.W.K.F. helped device characterization and J.H. revised the figures. X.X. and X.L. did the GI-XRD characterization. J.C. and F.Y. did the PL test. G.Z. and Y.Zhu finished the TEM characterization. G.L. K.L. and Y.Zhang prepared the manuscript. G.L., Y.Y. and K.L. supervised the execution of the project.

\section{Competing interests}

The authors declare no competing interests.

\section{Additional information}

Supplementary information The online version contains supplementary material available at https://doi.org/10.1038/s41467-021-25148-8.

Correspondence and requests for materials should be addressed to K.L. or G.L.

Peer review information Nature Communications thanks Alex Jen and the other, anonymous, reviewer(s) for their contribution to the peer review of this work.

Reprints and permission information is available at http://www.nature.com/reprints

Publisher's note Springer Nature remains neutral with regard to jurisdictional claims in published maps and institutional affiliations.

(c) (i) Open Access This article is licensed under a Creative Commons C. Attribution 4.0 International License, which permits use, sharing, adaptation, distribution and reproduction in any medium or format, as long as you give appropriate credit to the original author(s) and the source, provide a link to the Creative Commons license, and indicate if changes were made. The images or other third party material in this article are included in the article's Creative Commons license, unless indicated otherwise in a credit line to the material. If material is not included in the article's Creative Commons license and your intended use is not permitted by statutory regulation or exceeds the permitted use, you will need to obtain permission directly from the copyright holder. To view a copy of this license, visit http://creativecommons.org/ licenses/by/4.0/.

(C) The Author(s) 2021 